First publ. in: Physical Review E 55 (1997), 6, pp. 7153-7176

\title{
Asymptotic laws and preasymptotic correction formulas for the relaxation near glass-transition singularities
}

\author{
T. Franosch, M. Fuchs, W. Götze, M. R. Mayr, and A. P. Singh \\ Physik-Department, Technische Universität München, 85747 Garching, Germany
}

(Received 30 December 1996)

\begin{abstract}
Within the mode-coupling theory (MCT) for the dynamics of simple liquids, the leading corrections to the asymptotic solutions for the relaxation in the vicinity of an ideal glass transition are derived. The formulas are used to determine the range of validity of the scaling-law description of the MCT results for the $\alpha$ and $\beta$ processes in glass-forming systems. Solutions of the MCT equations of motion are calculated for a hard-sphere colloidal suspension model and compared with the derived analytical results. The leading-order formulas are shown to describe the major qualitative features of the bifurcation scenario near the transition and the leadingplus-next-to-leading-order formulas are demonstrated to give a quantitative description of the evolution of structural relaxation for the model. [S1063-651X(97)06005-4]
\end{abstract}

PACS number(s): 64.70.Pf, 61.20.Lc

\section{INTRODUCTION}

Glass-forming liquids develop structural-relaxation dynamics if they are cooled or compressed [1]. An outstanding feature of this relaxation is the stretching of decay functions $\Phi$ over large windows of time $t$, or equivalently, the stretching of spectra over huge windows of frequency $\omega$. Furthermore, the characteristic time scales $\tau$ for the dynamics can shift over several orders of magnitude if the temperature $T$ or the particle density $n$ are altered by, say, 20\%. The strong sensitivity of the scales $\tau$ on $T$ or $n$ is the origin of the glass transition at some temperature $T_{g}$ or density $n_{g}$, i.e., for the crossover from an equilibrium liquid to some nonequilibrium amorphous solid. Stretching was first reported by Kohlrausch [2] who described his dielectric relaxation data by the function $\Phi(t) \propto \exp -(t / \tau)^{\beta}, \beta<1$. von Schweidler [3] commented upon the fact, that certain parts of dielectric loss spectra vary according to the power law $\chi^{\prime \prime}(\omega) \propto 1 /(\omega \tau)^{b}, b<1$, if $\omega$ is shifted over two to three decades. Another fit formula for stretched susceptibilities was proposed by Cole and Cole [4]: $\chi(\omega) \propto 1 /\left[1+(-\mathrm{i} \omega \tau)^{a}\right], a<1$. It is an important issue of condensed matter physics to provide an understanding of structural-relaxation processes, in particular, of the mentioned anomalous exponents.

During recent years, the mode-coupling theory (MCT) has been developed as a model for the dynamics of strongly interacting disordered matter. MCT models are based on closed nonlinear integrodifferential equations for a set of correlation functions. The equations are regular, i.e., they depend smoothly on system parameters like $T$ and $n$; no assumptions on anomalous exponents, transitions or slow relaxations are built into the starting equations of the theory. The MCT yields the cited Kohlrausch formula [5], the von Schweidler function $[6,7]$, and the Cole-Cole susceptibility [8] as a description of their solutions in precisely defined asymptotic limits. Furthermore, MCT predicted a critical spectrum $\chi^{\prime \prime}(\omega) \propto \omega^{a}, 0<a<0.5$, which was experimentally detected in, for example, the molten salt $\mathrm{Ca}\left(\mathrm{NO}_{3}\right)_{2} \mathrm{KNO}_{3}$ (CKN) [9-11], the associated liquid glycerol [12-14], the covalently bonded system $\mathrm{B}_{2} \mathrm{O}_{3}$ [15], and in colloidal sus- pensions [16-21]. These mathematical and experimental findings appear as a justification to consider the MCT as a candidate for a theory of structural relaxation.

Originally, the MCT was proposed as a schematic treatment of glass transitions [22], as an approximation theory for the cage effect in simple liquids [23], and as a self-consistent one-loop approximation for a fluctuating-hydrodynamics model [24]. The new findings of the MCT result from a bifurcation singularity, which leads to an unconventional dynamics. Upon lowering $T$ or increasing $n$, the system is driven towards and past this singularity. Thereby, a scenario for the evolution of structural relaxation is obtained. For conventional glass-forming systems, the MCT predicts that structural-relaxation spectra evolve within the $\mathrm{GHz}$ band. This dynamical window is below the one studied for normal liquid motion but considerably above the window analyzed in the classical glass-transition research [1]. The GHz window became accessible only in recent years due to the invention of new spectrometers. The first complete documentation of the evolution of structural relaxation was obtained by $\mathrm{Li}$ et al. [11] for CKN and by van Megen and Underwood $[19,20]$ for a colloidal suspension. The former measured spectra as a function of temperature by depolarized light scattering, and the latter determined density fluctuation decay curves for various densities by photon correlation spectroscopy. The reported spectra and decay curves are strikingly similar to the corresponding results of MCT models. This observation was corroborated $[11,19,20]$ by detailed quantitative comparisons of the data with MCT formulas.

A variety of techniques has been used to explore the dynamics in windows relevant for a test of the MCTbifurcation predictions. Correlation functions $\Phi(t)$ have been measured by neutron-spin-echo spectroscopy [25], by stimulated Brillouin scattering [26], or by transient hole burning [27]. Computer simulations also yield $\Phi(t)$ as primary output, and recently Monte Carlo studies [28] and molecular-dynamics work $[29,30]$ could be extended to such long times, that they can deal with the same windows as achieved by the mentioned modern experiments. Inelasticlight scattering for frequencies between $0.1 \mathrm{GHz}$ and 10 
THz, pioneered by Cummins and co-workers [10-12,31,32], provides correlation spectra $\Phi^{\prime \prime}(\omega)$. So does inelastic neutron scattering, where great efforts have been made to extend the dynamical window to such sizes that the data become informative for glass-transition studies. This can be inferred from Refs. [9,13,33], to mention some particularly interesting examples. Dielectric loss studies deliver susceptibility spectra $\chi^{\prime \prime}(\omega)$, and this technique can now also be used in the $\mathrm{GHz}$ band $[34,35]$. Structural relaxation and the glass transition have also been studied for colloidal suspensions [16,18-21]. These systems are of particular relevance for tests of theories, because of the simplicity of their structure. From the cited papers one can infer that the MCT properly describes some features of the evolution of structural relaxation for some glass-forming systems; and this appears as a motivation to continue studies of the theory.

The MCT equations of motion are complicated and most of the theoretical work deals with attempts to understand the properties of their solutions. Up to now, leading-order asymptotic solutions have been worked out for parameters near the mentioned bifurcation singularity. This work, reviewed to a large extent in Refs. [36-38], established some universal features of the bifurcation scenario, and produced a series of handy formulas such as scaling laws. In principle, these formulas imply a series of relations between measurable quantities. It was suggested to use these results as a general basis for an assessment of the theory by experimental tests. Indeed, quite a number of papers have been published dealing with a comparison of experimental findings with leading-order asymptotic MCT results, which are partly reviewed in Refs. [37-39]. Recent work on the comparison of MCT results with light-scattering studies for conventional systems are reviewed in Refs. [40-42], on colloids in Refs. $[43,44]$, on neutron scattering in Ref. [45], and on computer simulations in Refs. [46-48]. In any experimental situation, the MCT analysis of the data is complicated by the fact, that there is no way to know a priori what the range of validity of the leading-order expansions should be. Therefore, it is timely to present a discussion of the asymptotic corrections, which lead to deviations from the widely used leading-order scaling laws.

The main aim of the present paper is the derivation of the next-to-leading corrections to the known, asymptotic results of the MCT. With these new results estimates of the range of validity of the leading asymptotic results become possible. For this second undertaking it is informative to compare the leading asymptotic results to numerical solutions of the MCT equations for a relevant model. Therefore, another goal of this paper is a comprehensive comparison of the leading asymptotic results with the MCT solutions for a liquid model. Thereto, in Sec. II, the MCT equations of motion for density fluctuations in simple liquids are specialized to a hard-sphere liquid model. The numerical solutions of this model serve as a basis for quantitative demonstrations of the analytical results. Section III presents the bifurcation in the equations for the long-time limits. Section IV introduces some concepts for the description of the bifurcation dynamics, and Sec. V discusses the two fractal power laws in time, which are the origin of the stretched decay in MCT. In Sec. VI the first scaling law is presented, which describes the relaxation in an intermediate time window. For longer times, a second scaling law describes the final relaxation into the liquid equilibrium, as shown in Sec. VII. We discuss the various qualitative trends of the deviations from the leading results. The analytical results for leading-plus-next-toleading expansions will also be compared with the mentioned solutions for the hard-sphere liquid model. The new results extend the range of validity of the old ones and provide a rather complete description of the bifurcation scenario for the model under discussion. Thus, the purpose of this paper is to establish an improved qualitative understanding of the MCT, to broaden the basis for experimental tests of the theory, and to deliver a set of reference results for future studies of other models.

\section{A MODE-COUPLING THEORY MODEL}

\section{A. The general equations of motion}

The basic version of the MCT deals with the dynamics by $M$ functions of time $t$, denoted by $\Phi_{q}(t), q=1, \ldots, M$. These functions obey the initial conditions $\Phi_{q}(0)=1, \partial_{t} \Phi_{q}(0)=0$, and their time evolution is determined by $M$ equations of motion

$$
\begin{array}{r}
\partial_{t}^{2} \Phi_{q}(t)+\Omega_{q}^{2} \Phi_{q}(t)+\int_{0}^{t}\left[M_{q}^{r e g}\left(t-t^{\prime}\right)\right. \\
\left.+\Omega_{q}^{2} m_{q}\left(t-t^{\prime}\right)\right] \partial_{t^{\prime}} \Phi_{q}\left(t^{\prime}\right) d t^{\prime}=0 .
\end{array}
$$

The model is specified by characteristic frequencies $\Omega_{q}>0$, by regular relaxation kernels $M_{q}^{r e g}(t)$, and by mode-coupling kernels $m_{q}(t)$. The latter are functions $\mathcal{F}_{q}$ of the $M$ variables $\Phi_{q}(t)$

$$
m_{q}(t)=\mathcal{F}_{q}(\Phi(t))
$$

These $\mathcal{F}_{q}$, which are called the mode-coupling functionals, are assumed to be absolutely monotone: $\mathcal{F}_{q}(f)$, as well as all its derivatives, are non-negative for $f_{q} \geqslant 0$. The functionals $\mathcal{F}_{q}(f)$ depend smoothly on control parameters such as $T$ or $n$. The $\Omega_{q}$ and $M_{q}^{r e g}(t)$ specify the transient dynamics; in particular, one gets $\Phi_{q}(t)=1-\left(\Omega_{q} t\right)^{2} / 2+O\left(t^{4}\right)$, if $M_{q}^{r e g}(t)$ is continuous in $t$. The interesting features of the MCT result from the interplay of nonlinearities, which are quantified by the kernels $m_{q}(t)$, with retardation phenomena, formulated by the convolution integral in Eq. (1).

In this paper the transient dynamics shall be simplified by two specializations. First, a Markovian model is used for the regular kernel: $M_{q}^{r e g}(t)=\nu_{q} \delta(t-0), \nu_{q}>0$. Second, the friction constants $\nu_{q}$ are assumed so large, that the inertia terms can be neglected. As a result, the generalized oscillator equations (1) are specialized to generalized relaxator equations

$\tau_{q} \partial_{t} \Phi_{q}(t)+\Phi_{q}(t)+\int_{0}^{t} m_{q}\left(t-t^{\prime}\right) \partial_{t^{\prime}} \Phi_{q}\left(t^{\prime}\right) d t^{\prime}=0$.

The transient dynamics is now quantified by the time constants $\tau_{q}=\nu_{q} / \Omega_{q}^{2}$. The initial conditions for the relaxator model have to be restricted to $\Phi_{q}(0)=1$. The relaxators ex- 
hibit the short-time asymptotes: $\Phi_{q}(t)=1-\left(t / \tau_{q}\right)+O\left(t^{2}\right)$. For vanishing kernels, $m_{q}=0$, the solutions describe uncoupled Debye relaxation processes: $\Phi_{q}^{(0)}(t)=\exp \left(-t / \tau_{q}\right)$.

One can show the following [49]: Eqs. (2), (3) define a unique solution. The solution is regular in the sense that all $M$ functions $\Phi_{q}(t)$ depend smoothly on $\tau_{q}$ and on smooth variations of $\mathcal{F}_{q}$ on any finite time interval. This means that the model is well defined; no ad hoc assumptions on singularities or glassy relaxations are made. Furthermore, the solutions can be written as superposition of Debye relaxation functions

$$
\Phi_{q}(t)=\int_{0}^{\infty} e^{-\gamma t} d \varrho_{q}(\gamma)
$$

where $\varrho_{q}(\gamma)$ is an increasing weight function. The Laplace transforms $\Phi_{q}(z)=\mathcal{L}\left[\Phi_{q}(t)\right](z)$ exist for complex frequencies $z, \operatorname{Im} z>0$. We use the convention $\mathcal{L}[f(t)](z)$ $=\mathrm{i} \int_{0}^{\infty} \exp (\mathrm{i} z t) f(t) d t$. For real frequencies $\omega$, one obtains with $z=\omega+\mathrm{i} 0: \Phi_{q}(z)=\Phi_{q}^{\prime}(\omega)+\mathrm{i} \Phi_{q}^{\prime \prime}(\omega)$, where $\Phi_{q}^{\prime}(\omega)=$ $\int_{0}^{\infty} \cos (\omega t) \Phi_{q}(t) d t>0$. The solutions $\Phi_{q}(t)$ have the properties of autocorrelation functions [50], and are therefore referred to as correlators. The $\Phi_{q}^{\prime \prime}(\omega)$ are called correlation spectra. In classical mechanics one can prove the fluctuationdissipation theorem which connects dynamical susceptibilities $\chi_{q}(\omega)$ and correlators [50]. We use the convention $\chi_{q}(\omega)=z \Phi_{q}(z)+1$ to define $\chi_{q}(\omega)$. In particular, the susceptibility spectra are given by $\chi_{q}^{\prime \prime}(\omega)=\omega \Phi_{q}^{\prime \prime}(\omega)$.

\section{B. A hard-sphere model}

An important quantity within the theory of simple classical $N$-particle liquids is the intermediate scattering function $F_{q}(t)$, i.e., the correlator for density fluctuations $\varrho_{\vec{q}}=N^{-1 / 2} \sum_{j} \exp \left(\overrightarrow{\mathrm{i}} \vec{q} \cdot \vec{r}_{j}\right)$ for wave vector $\vec{q}: F_{q}(t)$ $=\left\langle\varrho_{\vec{q}}(t)^{*} \varrho_{\vec{q}}\right\rangle$. The bracket denotes canonical averaging. These functions depend only on the wave-vector modulus $q=|\vec{q}|$. Their initial values are given by the static structure factor $S_{q}=\left\langle\left|\varrho_{q}\right|^{2}\right\rangle$, which can be expressed in terms of the direct correlation function $c_{q}$ via the Ornstein-Zernike formula: $S_{q}=1 /\left(1-n c_{q}\right)$. The characteristic frequencies $\Omega_{q}$ in Eq. (1), which determine the initial decay of $\Phi_{q}(t)=F_{q}(t) / S_{q}$, are given by $\Omega_{q}^{2}=(q v)^{2} / S_{q}$ with $v$ denoting the thermal velocity [51,52]. Equation (1) is an exact Zwanzig-Mori equation of motion relating the density correlators $\Phi_{q}(t)$ to the correlation functions of longitudinal fluctuating forces $M_{q}(t)=M_{q}^{r e g}(t)+\Omega_{q}^{2} m_{q}(t)$ [50-52]. The contribution $\Omega_{q}^{2} m_{q}(t)$ is due to coupling of forces to density fluctuation products, and all the rest is combined to $M_{q}^{r e g}(t)$. If one treats the correlations of density products with Kawasaki's factorization approximation $[53,54]$ one arrives at Eq. (2), where $\mathcal{F}_{q}$ is a quadratic polynomial

$$
\mathcal{F}_{q}(f)=\sum_{\vec{k}+\vec{p}=\vec{q}} V(\vec{q} ; \vec{k}, \vec{p}) f_{k} f_{p} .
$$

The non-negative coefficients $V$, the vertices, are equilibrium quantities [55]

$$
V(\vec{q} ; \vec{k}, \vec{p})=n S_{q} S_{k} S_{p}\left\{\vec{q}\left[\vec{k} c_{k}+\vec{p} c_{p}\right]\right\}^{2} /\left(2 q^{4}\right) .
$$

Formulas (1), (2), (5) define the simplified or ideal MCT [23]. They provide a self-consistent treatment of the cage effect. A review of the derivation, the contents, the shortcomings, and an extension of the ideal MCT can be found in Ref. [56].

The theory shall be specialized to a system of hard spheres with diameter $d$, whose equilibrium state is specified by a single control parameter, the packing fraction $\varphi=\pi n d^{3} / 6$. The structure factor shall be evaluated by the Percus-Yevick theory [52]. Thereby the $V(\vec{q} ; \vec{k}, \vec{p})$, and thus the functions $\mathcal{F}_{q}$, are obtained as smooth functions of $\varphi$. Furthermore, the wave-vector dependence of the regular friction constants shall be ignored, $\nu_{q}=\nu$; the time constants in the equations of motion are then given as smooth functions of $\varphi$ by

$$
\tau_{q}=t_{m i c} S_{q} /(q d)^{2}
$$

Here, $t_{m i c}=\nu(d / v)^{2}$ is a time scale for the dynamics on microscopic length scales. The sum in Eq. (5a) can be written as a double integral over $k=|\vec{k}|$ and $p=|\vec{q}-\vec{k}|$ which shall be approximated by a Riemann sum. To do this, a grid of 100 values for the moduli is chosen so that the $q d$ are placed with step size $h=0.4$ from 0.2 up to a cutoff $q^{*} d=39.8$,

$$
\begin{aligned}
\mathcal{F}_{q}(f)= & n\left[h^{3} /\left(32 d^{3} \pi^{2}\right)\right] \sum_{\hat{k}} \sum_{\hat{p}}{ }^{\prime} S_{q} S_{p} S_{k}\left(\hat{k} \hat{p} / \hat{q}^{5}\right) \\
& \times\left[\left(\hat{k}^{2}+\hat{q}^{2}-\hat{p}^{2}\right) c_{k}+\left(\hat{p}^{2}+\hat{q}^{2}-\hat{k}^{2}\right) c_{p}\right]^{2} f_{k} f_{p} .
\end{aligned}
$$

Here $\quad q d=h \hat{q}, k d=h \hat{k}, p d=h \hat{p} \quad$ and $\quad \hat{q}, \hat{k}, \hat{p}$ $=1 / 2,3 / 2, \ldots, 199 / 2$. The prime at the sum means that the summation is restricted to $|\hat{q}-\hat{k}|+1 / 2 \leqslant \hat{p} \leqslant \hat{q}+\hat{k}-1 / 2$. All numerical results and figures in this paper refer to the $M=100$ component model, defined by Eqs. (2), (3), (6), and (7). Units of length and time shall be chosen so that $d=1$ and $t_{m i c}=160$.

MCT equations for hard-sphere systems have already been studied before [23,57-60]. In the earlier work usually the Verlet-Weis approximation was used to evaluate $S_{q}$, the cutoff $q^{*}$ was chosen larger, the grid was taken finer, and special attempts were made to handle the small-wave-vector contributions to $\mathcal{F}_{q}$ carefully. Our simplifications lead to small differences of various amplitudes and constants compared to previous results. These differences do not exceed $5 \%$, and are therefore of no interest for the intention of this paper.

Equations (3) and (6) imply, that our model reproduces the initial decay of a hard-sphere colloidal suspension without hydrodynamic interaction [61]. It can therefore be considered as a model for a colloid where all interaction effects are ignored except those which describe the coupling between density fluctuation pairs and fluctuating forces. For such a model the MCT equations (2), (3), (5), and (6) can also be derived from the Smoluchowski equations $[62,63]$. 


\section{THE BIFURCATION OF THE GLASS-FORM FACTOR}

In this section some concepts and equations are introduced, which will be needed for a discussion of the MCT solutions near bifurcation points. The technique of asymptotic expansions is demonstrated for the form factor $f_{q}$ of the glass.

\section{A. The ideal liquid-glass transition}

Equation (4) shows that the correlators decrease monotonously towards their long-time limits $f_{q}=\Phi_{q}(t \rightarrow \infty)$, which obey $0 \leqslant f_{q}<1$. If these limits $f_{q}$ are zero, density fluctuations die out for long times, as one expects for an ergodic liquid. Edwards and Anderson pointed out in some other context [64], that a nontrivial $f_{q}>0$ is the signature of an ideal glass state. In this case, the dynamical structure factor $S_{q}(\omega)$ [50-52] exhibits an elastic contribution: $S_{q}(\omega)$ $=S_{q} \Phi_{q}^{\prime \prime}(\omega)=\pi f_{q} S_{q} \delta(\omega)+($ integrable function of $\omega$ ). Thus $f_{q}$-which is called Edwards-Anderson parameter, nonergodicity parameter, or glass-form factor-is the DebyeWaller factor of an ideal glass state.

From Eqs. (1) and (2) one derives $M$ coupled implicit equations for $f=\left(f_{1}, \ldots, f_{M}\right)$ [23]

$$
f_{q} /\left(1-f_{q}\right)=\mathcal{F}_{q}(f), \quad q=1, \ldots, M .
$$

The long-time limits $f_{q}$ obey the maximum property [36]: $\widetilde{f}_{q} \leqslant f_{q}, q=1, \ldots, M$, where the $\widetilde{f}_{q}$ abbreviate any solution of Eq. (8). Let us consider the sequence of vectors $f^{(j)}, j=0,1, \ldots$, defined by the iteration $f_{q}^{(j+1)} /$ $\left(1-f_{q}^{(j+1)}\right)=\mathcal{F}_{q}\left(f^{(j)}\right)$, starting with $f_{q}^{(0)}=1$. One can show [49] that the $f_{q}^{(j)}$ converge towards $f_{q}$ in the limit $j \rightarrow \infty$. We have used this iteration to evaluate $f_{q}$ for our model and found the critical packing fraction $\varphi_{c}=0.51591213$ (1) separating liquid from the glass solutions. Studies of glassy behavior require the analysis of dynamical windows and the shifts of spectral features over many orders of magnitude [1]. To reproduce such studies within the MCT one has to shift $\varphi-\varphi_{c}$ to very small values, as will be shown below in Figs. 4-6. To do this in a reproducible manner, one has to identify the critical point $\varphi_{c}$ with such high accuracy as noted.

For negative values of the reduced packing fraction $\epsilon=\left(\varphi-\varphi_{c}\right) / \varphi_{c}$, all correlators decay to zero, while for $\epsilon>0$ ideal glass states with $f_{q}>0$ are obtained. For $\varphi$ approaching the critical value from above, the $f_{q}$ approach positive constants, called critical form factors $f_{q}^{c}$. Considered as a function of $\varphi$, the long-time limits $f_{q}$ are discontinuous at $\varphi_{c}$. The point $\varphi=\varphi_{c}$ is also called glass-transition singularity; it marks a bifurcation point.

\section{B. The leading asymptotic results}

In order to study the form factor near the singularity we write $f_{q}=f_{q}^{c}+\left(1-f_{q}^{c}\right)^{2} g_{q}$ and solve Eqs. (8) for small $g_{q}$ and small positive $\epsilon$. The leading term of this expansion has already been worked out in Ref. [7], but we need to recall some of the earlier results as basis of the intended extensions of the analytical work. The nontrivial bits of the expansion are the Taylor coefficients of $\mathcal{F}_{q}$ for $f=f^{c}$, which shall be used with the convention

$$
\begin{aligned}
C_{q k_{1} \cdots k_{m}}= & \frac{1}{m !}\left[\partial^{m} \mathcal{F}_{q}\left(f^{c}\right) / \partial f_{k_{1}} \cdots \partial f_{k_{m}}\right] \\
& \times\left(1-f_{k_{1}}^{c}\right)^{2} \cdots\left(1-f_{k_{m}}^{c}\right)^{2} .
\end{aligned}
$$

These are smooth positive functions of $\epsilon$. The values of these and other quantities for $\epsilon \rightarrow+0$ shall be indicated by a superscript $c$. Let us introduce the differences $\Delta C=C-C^{c}$, so that

$$
\begin{gathered}
C_{q k_{1} \cdots k_{m}}=C_{q k_{1} \cdots k_{m}}^{c}+\Delta C_{q k_{1} \cdots k_{m}}, \\
\Delta C_{q k_{1} \cdots k_{m}}=\epsilon C_{q k_{1} \cdots k_{m}}^{\prime c}+O\left(\epsilon^{2}\right) .
\end{gathered}
$$

One can rewrite Eq. (8) as

$$
\sum_{k=1}^{M}\left[\delta_{q k}-C_{q k}^{c}\right] g_{k}=I_{q} .
$$

This result looks like a set of $M$ linear equations. However, the nonlinearities are hidden in the inhomogeneity $I_{q}$, which shall be written as a sum of two terms

$$
\begin{gathered}
I_{q}=I_{q}^{(1)}+I_{q}^{(2)}, \\
I_{q}^{(1)}=\Delta C_{q}-\left(1-f_{q}^{c}\right) g_{q}^{2}+\sum_{k p} C_{q k p}^{c} g_{k} g_{p}, \\
I_{q}^{(2)}=\sum_{k} \Delta C_{q k} g_{k}-\left(1-f_{q}^{c}\right)^{2} g_{q}^{3}+\sum_{k p l} C_{q k p l}^{c} g_{k} g_{p} g_{l} \\
+O\left(\epsilon g^{2}, g^{4}\right) .
\end{gathered}
$$

Let us also consider the matrix $\hat{C}_{q k}$ $=\left[\partial \mathcal{F}_{q}(f) / \partial f_{k}\right]\left(1-f_{k}\right)^{2}$. The Jacobian matrix of the system of equations (8) is equivalent to the $M \times M$ matrix $1-\hat{C}$. The matrix $\hat{C}$ has only non-negative elements: $\hat{C}_{q k} \geqslant 0$. Thus, according to the Frobenius-Perron theorems [65], there is a nondegenerate maximum eigenvalue $E$ of the matrix $\hat{C}$; generically any other eigenvalue, say $E^{\prime}$, obeys $\left|E^{\prime}\right|<E$. One can show [49] that $E \leqslant 1$. If $E<1$, the Jacobian matrix can be inverted and $f$ varies smoothly with $\varphi$. The condition for a glass-transition singularity therefore is $E=E^{c}=1$. For $\varphi=\varphi_{c}$, one gets $\hat{C}_{q k}=C_{q k}^{c}$. The right and left eigenvectors of $C^{c}$ belonging to the eigenvalue unity shall be denoted by $e$ and $\hat{e}$, respectively,

$$
\sum_{k} C_{q k}^{c} e_{k}=e_{q} ; \quad \sum_{q} \hat{e}_{q} C_{q k}^{c}=\hat{e}_{k}
$$

Generically, the numbers $e_{q}, \hat{e}_{q}$ can be chosen positive [65]. To fix the vectors uniquely we impose the convention: $\Sigma_{q} \hat{e}_{q} e_{q}=1$ and $\Sigma_{q} \hat{e}_{q}\left(1-f_{q}^{c}\right) e_{q}^{2}=1$.

Due to the vanishing of the determinant of the matrix $1-C^{c}$, Eq. (10a) can only be solved if the inhomogeneity on the right-hand side satisfies the solubility condition

$$
\sum_{q} \hat{e}_{q} I_{q}=0
$$


The general solution of Eq. (10a) is the sum of a multiple of the dangerous eigenvector, say $g e$, and a special solution, say $\widetilde{g}$. The latter shall be fixed uniquely by the condition $\Sigma_{q} \hat{e}_{q} \widetilde{g}_{q}=0$. It can be written as a linear combination of the inhomogeneities

$$
g_{q}=g e_{q}+\widetilde{g}_{q}, \quad \widetilde{g_{q}}=\sum_{p} R_{q p} I_{p} .
$$

One can use simple series expansions [36] to evaluate $E, \hat{e}, e$, and $R$.

The glass-transition singularity is caused by the Jacobian matrix to have a vanishing nondegenerate eigenvalue $1-E$. This means that the MCT bifurcation is a cuspoid bifurcation $A_{l}, l=2,3, \ldots$ in Arnold's terminology [66]. For $\varphi \rightarrow \varphi_{c}$, $l$ solutions of Eq. (8) coalesce. The simplest possibility, the Whitney fold bifurcation $A_{2}$, occurs if the positive number $\lambda=\Sigma_{q k p} \hat{e}_{q} C_{q k p}^{c} e_{k} e_{p}$ differs from unity and then the solution for small $\epsilon>0$ can be obtained by an expansion in powers of $\sqrt{\epsilon}$. In this case one gets $I^{(2)}=O\left(\epsilon^{3 / 2}\right)$, $g=O(\sqrt{\epsilon}), \tilde{g}=O(\epsilon)$. Thus, the solubility condition (12a) reads in leading order $\Sigma_{q} \hat{e}_{q} I_{q}^{(1)}=0$ and $I^{(1)}$ can be obtained from Eq. (10c) with $g_{q}=g e_{q}$. One finds $\sigma-g^{2}+\lambda g^{2}=0$. We get for our model $\lambda=0.735$. The quantity $\sigma=\Sigma_{q} \hat{e}_{q} \Delta C_{q}$, which is called the separation parameter, varies smoothly with $\epsilon$. In leading order in $\epsilon$ one can write

$$
\sigma=C \epsilon, \quad C=\sum_{q} \hat{e}_{q} C_{q}^{\prime c},
$$

where for our model $C=1.54$. Thus one finds $g= \pm \sqrt{\sigma /(1-\lambda)}+O(\epsilon)$ for $\sigma>0$. Because of the maximum property the positive solution for $g$ describes the small $\epsilon$ asymptotics of the glass-form factor. Introducing the critical amplitude $h_{q} \geqslant 0$ by

$$
h_{q}=\left(1-f_{q}^{c}\right)^{2} e_{q},
$$

the leading-order result can therefore be noted as

$$
f_{q}=f_{q}^{c}+h_{q} \sqrt{\sigma /(1-\lambda)}, \quad \sigma>0 .
$$

Figure 1 exhibits the separation parameter $\sigma$ as a function of the packing fraction $\varphi$ in comparison with the linear asymptote (13). The latter describes $\sigma$ correctly within $10 \%$ for $|\epsilon| \leqslant 0.04$. The next Taylor expansion term of $\sigma$ is of order $\epsilon^{2}$ and, therefore, it would influence the asymptotic expansion of $f$ as a correction of order $\epsilon^{3 / 2}$. Since we are going to consider only leading and next-to-leading expansions in $\sqrt{\epsilon}$, the $\epsilon^{2}$ correction of $\sigma$ does not enter. Therefore, throughout the rest of this paper, $\sigma$ is merely meant to abbreviate $C \epsilon$.

In Fig. 2(a), the structure factor $S_{q}$ is shown for $\epsilon=0$ and $\epsilon= \pm 10^{-4 / 3} . S_{q}$ is the basic input for our model. In Fig. 2(b), the results for the critical form factor $f_{q}^{c}$ and the critical amplitude $h_{q}$ are shown. For large $S_{q}$, the compressibility $\kappa_{q}$ $\propto S_{q}$ is large. Therefore spontaneous arrest is easy, and $f_{q}^{c}$ exhibits a maximum near the position of the structure-factor peak; with varying $q, f_{q}^{c}$ oscillates in phase with $S_{q}$. Since $f_{q} \leqslant 1, f_{q}-f_{q}^{c}$ is bounded by $1-f_{q}^{c}$. The amplitude $h_{q}$ for

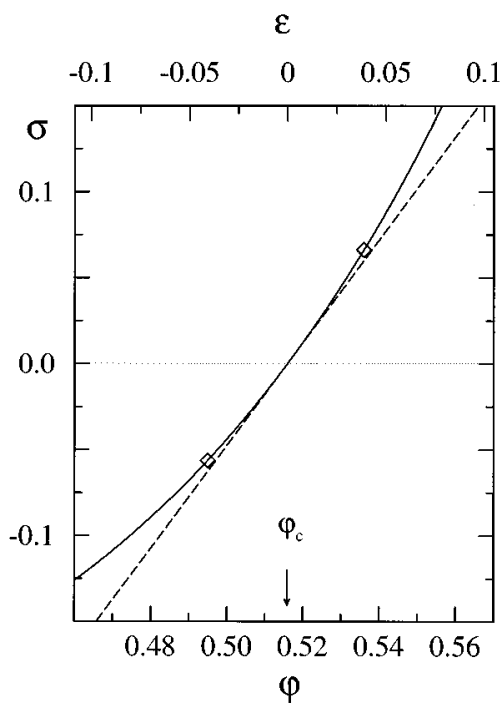

FIG. 1. The separation parameter $\sigma$ as function of the packing fraction $\varphi$ (solid line). The dashed line is the linear asymptote $\sigma=1.54 \epsilon, \epsilon=\left(\varphi-\varphi_{c}\right) / \varphi_{c}, \varphi_{c} \approx 0.516$. The diamonds mark the values $\varphi=0.495$ and 0.536 , where the asymptote deviates from $\sigma$ by $10 \%$.

the increase of $f_{q}$ is therefore much smaller for $q \sim 7$ than for $q$ off the structure-factor-peak position. The transition is driven primarily by the wave-vector contributions from $q \sim 7$. For very large $q$, both $f_{q}^{c}$ and $h_{q}$ become small.

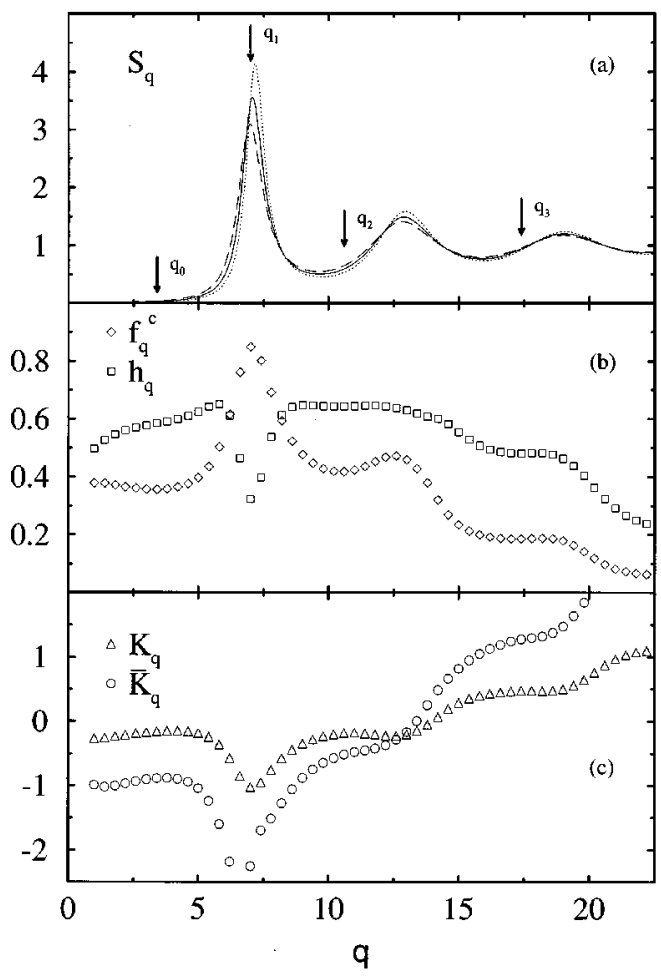

FIG. 2. (a) Structure factor $S_{q}$ as function of wave vector $q$ for $\varphi=\varphi_{c} \approx 0.516$ (solid curve), $\varphi=0.492$ (dashed curve), and $\varphi=0.540$ (dotted curve). The arrows mark the wave vectors $q_{0}=3.4, q_{1}=7.0, q_{2}=10.6$, and $q_{3}=17.4$. (b) The critical form factor $f_{q}^{c}$ (diamonds) and the critical amplitude $h_{q}$ (squares). (c) The amplitudes $\bar{K}_{q}$ [Eq. (16), circles] and $K_{q}$ [Eq. (23), triangles]. 


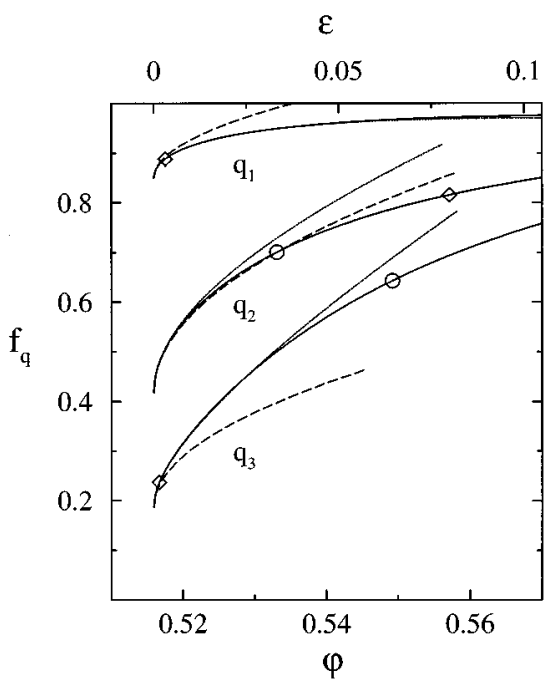

FIG. 3. Glass-form factors $f_{q}$ for the wave vectors $q_{1}=7.0$, $q_{2}=10.6$, and $q_{3}=17.4$ (solid lines). The leading asymptotes [Eq. (16), dashed] describe the result $f_{q}-f_{q}^{c}$ within $10 \%$ up to the packing fractions which are marked by diamonds. The formula including the next-to-leading asymptote [Eq. (18), dotted] works within a $10 \%$ accuracy level up to $\epsilon=0.141,0.033,0.064$ for $q_{1}, q_{2}, q_{3}$, respectively; the corresponding packing fractions are marked by circles.

In the following, the dynamics will be discussed in detail for the two representative wave vectors $q_{1}=7.0, q_{2}=10.6$. The first one is close to the position of the structure-factor peak and the second one is close to the first minimum position [compare Fig. 2(a)]. These vectors will be referred to as 1 and 2 . One gets $S_{1}^{c}=3.49, S_{2}^{c}=0.597 ; f_{1}^{c}=0.849, f_{2}^{c}$ $=0.417 ; h_{1}=0.323, h_{2}=0.642$. Let us also note the time scales for the transient motion, Eq. (6): $\tau_{1}^{c}=11.4, \tau_{2}^{c}=0.851$. In Fig. 3, the variation of $f_{q}$ with changes of the density is compared with the leading asymptotic formula (15). It works on a $10 \%$ accuracy level for $q_{1}$ up to $\epsilon=0.003$ only. The comparison for the large wave vector $q_{3}=17.4$ is also shown, where a $10 \%$ accurate description can be achieved only for $\epsilon \leqslant 0.002$. For $q_{2}$ the $10 \%$ approximation works up to $\epsilon=0.08$, but this large value is mainly due to an accidental cancelation of higher-order terms.

\section{The leading corrections}

There are two contributions to the next-to-leading order of the expansion of $g_{q}$. One is given by $\widetilde{g_{q}}$ in Eq. (12b). It is obtained from $I_{q}$ which can be approximated by $I_{q}^{(1)}$. In the leading part $I_{q}^{(1)}$ of the inhomogeneity, Eq. (10c), one can substitute $\Delta C_{q}=C_{q}^{\prime c} \epsilon$ and $g_{q}=e_{q} \sqrt{\sigma /(1-\lambda)}$. The result shall be written as $\widetilde{g}_{q}=e_{q} \sigma \bar{K}_{q} / \sqrt{1-\lambda}$, so that

$$
\begin{aligned}
\bar{K}_{q}= & \sum_{k} R_{q k}\left\{\sqrt{1-\lambda} C_{k}^{\prime c} / C-\left[\left(1-f_{k}^{c}\right) e_{k}^{2}\right.\right. \\
& \left.\left.-\sum_{p l} C_{k p l}^{c} e_{p} e_{l}\right] / \sqrt{1-\lambda}\right\} / e_{q} .
\end{aligned}
$$

The other contribution is obtained by solving Eq. (12a) with the formula $g=\sqrt{\sigma /(1-\lambda)}[1+\kappa \sqrt{\sigma}+O(\sigma)]$ in Eq. (12b). For the coefficient $\kappa$, one finds

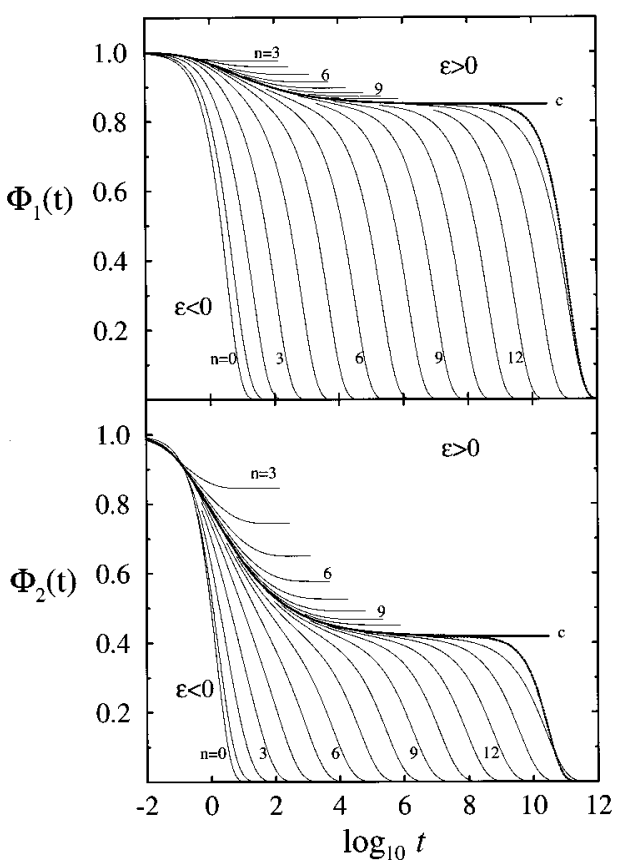

FIG. 4. Correlators $\Phi_{1}$ and $\Phi_{2}$ for the two wave vectors $q_{1}=7.0$ and $q_{2}=10.6$, respectively, as function of $\log _{10} t$, calculated for the packing fractions $\varphi=\varphi_{c}(1+\epsilon), \epsilon= \pm 10^{-n / 3}$. The thick curves labeled $c$ are the solutions for $\epsilon=0$. The dotted lines are Debye functions $f^{c} \exp -\left(t / \tau_{D}\right)$ with $f^{c}=0.849, \tau_{D}=1.37 \times 10^{11}$, and $f^{c}=0.417, \tau_{D}=2.83 \times 10^{10}$ for $q_{1}$ and $q_{2}$, respectively.

$$
\begin{aligned}
\kappa= & \frac{1}{1-\lambda} \sum_{q} \hat{e}_{q}\left\{\sum_{k p} C_{q k p}^{c} e_{k} e_{p} \bar{K}_{p}-\left(1-f_{q}^{c}\right) e_{q}^{2} \bar{K}_{q}\right. \\
& +\frac{\sqrt{1-\lambda}}{2} \sum_{k} C_{q k}^{\prime c} e_{k} / C-\frac{1}{2 \sqrt{1-\lambda}}\left[\left(1-f_{q}^{c}\right)^{2} e_{q}^{3}\right. \\
& \left.\left.-\sum_{k p l} C_{q k p l}^{c} e_{k} e_{p} e_{l}\right]\right\} .
\end{aligned}
$$

For our model, one gets $\kappa=0.961, \quad \bar{K}_{1}=-2.26, \quad \bar{K}_{2}$ $=-0.515, \bar{K}_{3}=1.28$. The desired result, which describes $f_{q}$ up to errors of order $\epsilon^{3 / 2}$, reads

$$
f_{q}-f_{q}^{c}=h_{q} \sqrt{\sigma /(1-\lambda)}\left[1+\sqrt{\sigma}\left(\bar{K}_{q}+\kappa\right)\right] .
$$

The leading-order result (15) describes $f_{q}-f_{q}^{c}$ within a small relative error $\epsilon^{*}$ as long as the separation parameter obeys the inequality $\sigma \leqslant \epsilon^{* 2} /\left(\bar{K}_{q}+\kappa\right)^{2}$. Figure 2(c) shows the amplitude $\bar{K}_{q}$ for our model. In Fig. 3 it is demonstrated that the result (18) describes the solution on a $10 \%$ accuracy level for $q_{1}\left(q_{2}, q_{3}\right)$ up to the packing fractions $\varphi=0.589$ $(0.533,0.549)$, respectively.

\section{THE TWO-STEP-RELAXATION SCENARIO}

Figures 4-6 demonstrate the dynamics of our model. The curves labeled $c$ exhibit the critical dynamics, i.e., they are calculated for $\varphi=\varphi_{c}$. The others refer to reduced packing fractions, which are spaced with equal distance on a logarithmic scale with three values per decade: $\epsilon= \pm 10^{-n / 3}$, 


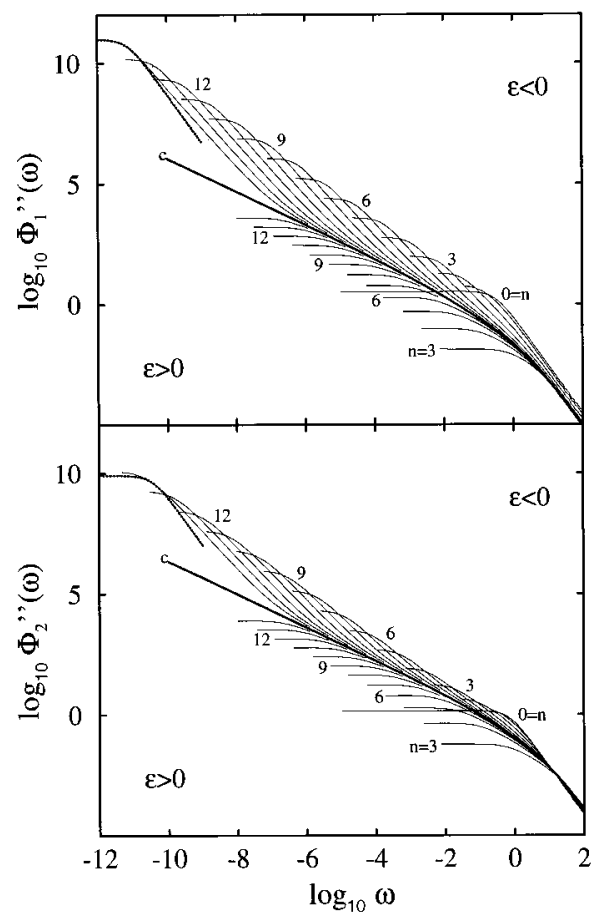

FIG. 5. Correlation spectra $\Phi_{1}^{\prime \prime}(\omega)$ and $\Phi_{2}^{\prime \prime}(\omega)$ as function of frequency $\omega$ for the results shown in Fig. 4. The dotted lines are Debye spectra $\Phi_{D}(\omega)=2 \chi_{\max } \tau_{D} /\left[1+\left(\omega \tau_{D}\right)^{2}\right]$, with $\chi_{\max }$ $=0.370$ for $q_{1}$ and $\chi_{\max }=0.147$ for $q_{2}$, and $\tau_{D}$, specified in connection with Fig. 4.

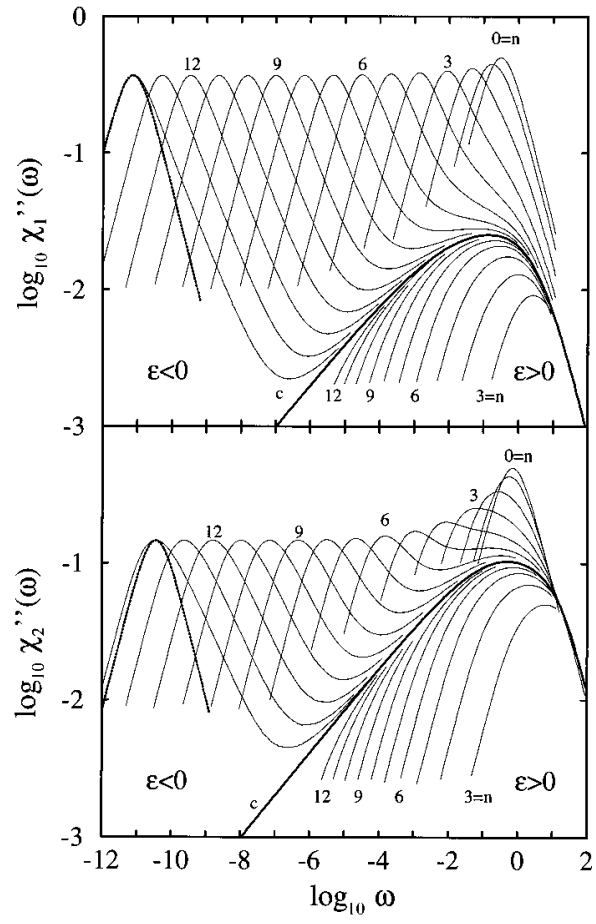

FIG. 6. Susceptibility spectra $\chi^{\prime \prime}(\omega)=\omega \Phi^{\prime \prime}(\omega)$ for the results shown in Figs. 4 and 5. The dotted lines are Debye peaks $\chi_{D}^{\prime \prime}(\omega)=2 \chi_{\max } \omega \tau_{D} /\left[1+\left(\omega \tau_{D}\right)^{2}\right]$, with $\tau_{D}$ and $\chi_{\max }$ specified in connection with Figs. 4 and 5 , respectively. $n=0,1, \ldots, 14$. Results for $\epsilon>0$ and $n=0,1,2$ are not considered, since for the corresponding large packing fractions the Percus-Yevick theory yields unphysical negative pairdistribution functions. Notice, that the curves for $\epsilon<0$ and $n=0$ refer to a vanishing relaxation kernel; they demonstrate elementary dynamics, as described by the Debye law. In this section some general features of the shown transition scenario shall be pointed out, and some concepts for its description shall be introduced.

The correlators $\Phi_{q}(t)$ are smooth functions of $\epsilon$ on any finite interval of time [49]. Therefore they have to be close to the critical correlators $\Phi_{q}^{c}(t)$ if $|\epsilon|$ is small and if the time is smaller than some crossover time $\tau_{c o}$. The cited smoothness implies that this time scale $\tau_{c o}$ diverges if the critical point is approached: $\tau_{c o}(\epsilon \rightarrow 0) \rightarrow \infty$. There appears a first window for stretched relaxation. It deals with the dynamics for times which are larger than some time $t_{0}$ characterizing the transient but which are short compared to $\tau_{c o}$. The first relaxation step describes the long-time decay towards the critical plateau $f_{q}^{c}$. For $t \ll \tau_{c o}$, the decay curves are $\epsilon$ insensitive as is demonstrated in Fig. 4. For normal liquids, one finds a white-noise spectrum, $\Phi_{\text {white noise }}^{\prime \prime}(\omega) \propto \omega^{0}$, for frequencies $\omega$ below the band characterizing microscopic motion $[51,52]$. This behavior is demonstrated in Fig. 5 by the Debye spectra (the curves for $\epsilon<0, n=0$ ) for $\omega<0.1$. The stretching of the critical dynamics implies an enhancement of the spectra $\Phi_{q}^{\prime \prime}(\omega)$ above the mentioned white-noise level for $1 / \tau_{c o} \ll \omega \ll 1 / t_{0}$. For $q=q_{2}$ and $n \geqslant 10$, one infers from Fig. 5 that the critical-spectrum enhancement amounts to more than a factor 100 if $\omega$ decreases three decades below 0.1 . A white-noise spectrum leads to a linear susceptibility spectrum, $\chi_{\text {white noise }}^{\prime \prime}(\omega) \propto \omega$, as is demonstrated in Fig. 6 for the $\epsilon<0, n=0$ results for $\omega<0.1$. The spectral enhancement due to the critical dynamics leads to a sublinear susceptibility variation for small frequencies $\chi_{q}^{\prime \prime}(\omega) \propto \omega^{a}, a<1$, within the window $1 / \tau_{c o} \ll \omega \ll 1 / t_{0}$.

Let us define the crossover time scale $\tau_{c o}$ more precisely. For the liquid, the scale is denoted by $\tau_{q}^{-}$; it shall be chosen as the one, where the critical plateau is crossed: $\Phi_{q}\left(\tau_{q}^{-}\right)=f_{q}^{c}$. For the glass, the scale is denoted by $\tau_{q}^{+}$; it shall be taken as the one where the plateau is reached within 0.1\%: $\Phi_{q}\left(\tau_{q}^{+}\right)-f_{q}^{c}=1.001\left(f_{q}-f_{q}^{c}\right)$. Figure 7(a) exhibits these scales as a function of $\varphi$ for $q=q_{2}$. One can check that for small $|\epsilon|$ the ratio $\tau_{q}^{-}(-|\epsilon|) / \tau_{q}^{+}(|\epsilon|)$ becomes $\epsilon$ independent. In Fig. 7(c), the ratio $\tau_{1}^{ \pm} / \tau_{2}^{ \pm}$of the scales for the two wave vectors $q_{1}, q_{2}$ are shown as a function of $\varphi$. For small $|\epsilon|$, the ratios are unity, i.e., the scales are asymptotically independent of $q$. In this sense one concludes, that there is only one scale $\tau_{c o}$, terminating the first structural-relaxation step. The divergence of $\tau_{c o} \propto \tau_{q}^{+}$for $\varphi \rightarrow \varphi_{c}+0$ is the signature of the glass instability at the transition point. The divergence of $\tau_{c o} \propto \tau_{q}^{-}$for $\varphi \rightarrow \varphi_{c}-0$ signalizes the freezing of the liquid at the critical point.

The liquid solutions exhibit a second relaxation process for $t>\tau_{q}^{-}$. It deals with the decay from the critical plateau $f_{q}^{c}$ to zero. The second relaxation step leads to the $\epsilon$ sensitive quasielastic peaks of the relaxation spectra in Fig. 5 and to the corresponding low-frequency peaks for the susceptibility spectra in Fig. 6. These peaks are the analog of Mountain's 


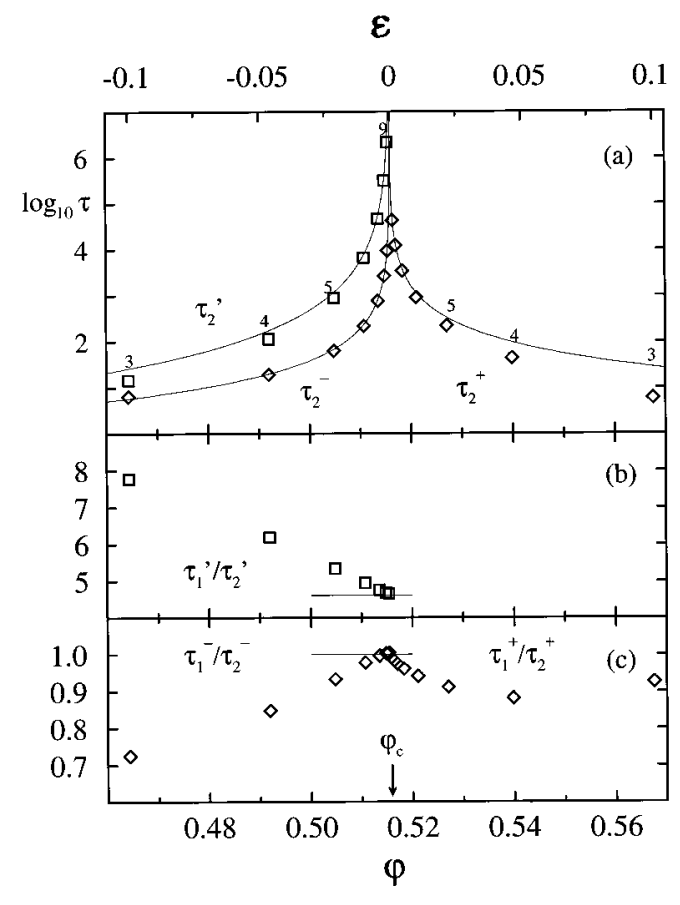

FIG. 7. (a) The crossover times $\tau_{q}^{\mp}$ (diamonds) characterizing the first relaxation step and the $\alpha$-relaxation time $\tau_{q}^{\prime}$ (squares) as a function of $\varphi$ for the reduced packing fraction $\epsilon=\mp 10^{-n / 3}, n=$ 3-9 for the wave vector $q_{2}=10.6$. The solid lines are the powerlaw functions: $\tau^{\mp}=c_{\mp} /|\epsilon|^{\delta}, c_{-}=0.149, c_{+}=0.706, \delta=1.60, \tau^{\prime}=$ $c^{\prime} /|\epsilon|^{\gamma}, c^{\prime}=0.0920, \gamma=2.46$. (b) Ratio of the $\alpha$-relaxation scales $\tau_{1}^{\prime} / \tau_{2}^{\prime}$ (squares) for wave vectors $q_{1}=7.0$ and $q_{2}=10.6$. (c) The ratio of the crossover scales $\tau_{1}^{ \pm} / \tau_{2}^{ \pm}$(diamonds) for the wave vectors $q_{1}, q_{2}$. The horizontal lines in (b) and (c) mark the $\varphi \rightarrow \varphi_{c}$ asymptotes 4.6 and 1.0 , respectively.

peaks [67] for molecular liquids. Within the MCT for simple liquids, it is the whole cluster of a particle with its cage which plays the role of the molecule. Following the terminology of the glass literature [1], the second relaxation step is called the $\alpha$ process.

An $\alpha$-relaxation time scale $\tau_{q}^{\prime}$ can be defined, for example, as $1 / \tau_{q}^{\prime}=\omega_{\max }(q)$, where $\omega_{\max }(q)$ denotes the position of the $\alpha$-peak maximum of the susceptibility spectra. Again, one concludes from the convergence $\Phi_{q}(t) \rightarrow \Phi_{q}^{c}(t)$, for $\epsilon \rightarrow 0$ and for any fixed finite time interval, that $\tau_{q}^{\prime} \rightarrow \infty$ for $\epsilon \rightarrow 0-$. Figure 7(a) exhibits the strong increase of $\tau_{q}^{\prime}$ upon approaching the bifurcation point for $q=q_{2}$. Another possibility for defining an $\alpha$ scale, say $\vec{\tau}_{q}^{\prime}$, is given by $\Phi_{q}\left(\vec{\tau}_{q}\right)=f_{q}^{c} / 2$. One deduces from Figs. 4 and 6, however, that $\tau_{q}^{\prime} / \vec{\tau}_{q}$ are independent of $\epsilon$ for small separations. The $\alpha$ scales for different correlators are different as one infers by comparing Fig. 4(a) with 4(b) or Fig. 6(a) with 6(b). However, the ratio of the scales, say $\tau_{1}^{\prime} / \tau_{2}^{\prime}$, becomes independent of $\epsilon$ for $\varphi \rightarrow \varphi_{c}-0$ as is shown in Fig. 7(b). Both scales diverge for $\epsilon \rightarrow 0$ but so that $\tau_{1}^{\prime}=C_{1} \tau^{\prime}$ and $\tau_{2}^{\prime}=C_{2} \tau^{\prime}$, where $\tau^{\prime} \rightarrow \infty$ for $\epsilon \rightarrow 0-$, and $C_{1,2}$ asymptotically are $\epsilon$ independent. In this sense one concludes, that the $\alpha$ scales of different correlators are coupled, to use another concept from the glass literature [1]. Asymptotically, the slowing down of the $\alpha$ process is characterized by a single scale $\tau^{\prime}$.
Upon approaching the transition, the length of the time interval where $\Phi_{q}(t)$ is close to the critical plateau $f_{q}^{c}$ increases, as is obvious from Fig. 4. For $\epsilon \rightarrow 0-$, both characteristic times $\tau^{-}$and $\tau^{\prime}$ become large relative to the microscopic time scale $t_{0}$. However, the second time scale becomes large even relative to the large scale $\tau^{-}$of the first process: $\tau^{\prime} / \tau^{-}$increases for $\epsilon \rightarrow 0-$. This is demonstrated in Fig. 7(a). The curves for $n$ and $n+1$ in Figs. 4-6 differ in factors $10^{-1 / 3}$ for the reduced packing fraction $\epsilon$. One infers from Fig. 4 that the corresponding times $\vec{\tau}_{q}$ also differ by a constant factor $x$. Similarly Fig. 6 demonstrates that the $\omega_{\max }(q)$ differ by the same factor $x$. This means, that the $\alpha$-relaxation scale follows a power law: $\tau^{\prime} \propto 1 /|\epsilon|^{\gamma} ; \gamma$ $=3 \log _{10} x$. The analogous conclusion is reached for the first relaxation scale: $\tau_{q}^{ \pm} \propto 1 /|\epsilon|^{\delta}$. The found increase of $\tau^{\prime} / \tau^{-}$is equivalent to $\gamma>\delta$. Power-law functions are shown as lines in Fig. 7(a).

The $\alpha$ spectrum of $\Phi_{q}^{\prime \prime}(\omega)$ is not placed on top of some white-noise background but on top of an anomalous spectrum. It is easier to avoid mixing up the two spectra due to the two relaxation steps, if one considers the susceptibility spectra of Fig. 6. The high-frequency wing of the $\alpha$ peak is a function, which decreases with increasing $\omega$. But the critical susceptibility spectrum increases with $\omega$. Thus the crossover from the first to the second relaxation step manifests itself in a minimum at some frequency $\omega_{\min }(q)$. The critical spectrum leads to an enhancement of the spectrum $\chi_{\min }(q)=\chi_{q}^{\prime \prime}\left(\omega_{\min }(q)\right)$ above a white-noise background: $\chi_{\text {min }}(q) \gg \chi_{\text {white noise }}^{\prime \prime}\left(\omega_{\min }(q)\right)$. The first step of the structural relaxation of the liquid leads to the spectrum within the window $\omega_{\min }(q) \ll \omega \ll 1 / \tau_{q}$. The second step of the structural relaxation leads to the low-frequency spectra for $\omega \ll \omega_{\min }(q)$.

The glass correlators do not exhibit a second relaxation step, rather they arrest at $f_{q}>f_{q}^{c}$ for $t \gg \tau_{c o}$. If one wanted to characterize the long-time dynamics of the glass also by a time scale $\tau^{\prime}$, one would have to use $1 / \tau^{\prime}=0$. For $\varphi \neq \varphi_{c}$ the distribution of rates in Eq. (4) exhibits a gap [49]. This means that all correlators approach their long-time limit exponentially for $\epsilon \neq 0$. Therefore the glass spectra vary regularly for $\omega \ll 1 / \tau_{c o}$. The crossover from the linear lowfrequency spectrum, $\chi_{q}^{\prime \prime}\left(\omega \ll 1 / \tau_{c o}\right) \propto \omega \tau_{q}^{+}$, to the sublinear critical spectrum $\chi_{q}^{\prime \prime}\left(\omega \gg 1 / \tau_{c o}\right) \propto\left(\omega t_{0}\right)^{a}$, produces a knee at some frequency $\omega_{K}(q)$. The position of the knee can be defined, for example, as the one of the maximum of the $\chi_{q}^{\prime \prime}(\omega) / \sqrt{\omega}$ versus $\omega$ curve. Again the knee intensity $\chi_{K}(q)=\chi_{q}^{\prime \prime}\left(\omega_{K}(q)\right)$ is enhanced above the white noise: $\chi_{K}(q) \gg \chi_{\text {white noise }}^{\prime \prime}\left(\omega_{K}(q)\right)$. By constructing similar figures as Fig. 7 one can convince oneself that $\omega_{\min }(q) \propto 1 / \tau_{q}^{-}$and $\omega_{K}(q) \propto 1 / \tau_{q}^{+}$.

For a Debye correlator, the time ratio $\left(t / \tau_{q}\right)$ has to increase by about 1.34 decades from 0.105 to 2.303 in order to scan the decay from $90 \%$ to $10 \%$ of the initial value. For the same decay, the correlator $\Phi_{2}$ for $\epsilon<0, n=6$ requires an increase of $t$ by about a factor $6.33 \times 10^{4}$ as one infers from Fig. 4(b). This stretching of the specified time interval over more than four orders of magnitude is equivalent to a stretching of the susceptibility spectrum over a corresponding huge frequency window. A Debye susceptibility peak 
$\chi_{D}^{\prime \prime}(\omega)=2 \chi_{\max } \omega \tau_{D} /\left[1+\left(\omega \tau_{D}\right)^{2}\right]$ has a width at half maximum of 1.14 decades. The spectrum $\epsilon<0, n=6$ in Fig. 6(b) extends at half height from $\omega=2.81 \times 10^{-5}$ to $\omega=5.61$. Its width is more than 14000 times larger than that for a Debye process.

Figure 6 exhibits Debye peaks as dotted lines; $\tau_{D}=\tau^{\prime}$ and the $\chi_{\max }$ are adjusted so that the $\alpha$-peak maxima for the $\epsilon<0, n=14$ results are matched. The corresponding correlation spectra $\Phi_{D}^{\prime \prime}(\omega)=\chi_{D}^{\prime \prime}(\omega) / \omega$ are shown in Fig. 5 and the decay curves $f_{q}^{c} \exp \left(-t / \tau_{D}\right)$ are included as dotted lines in Fig. 4. Comparing the dotted lines with the ones for the $n=14 \alpha$ process, one concludes that stretching is not only due to the crossover from the first to the second relaxation step, as discussed in the preceding paragraph. The $\alpha$ process itself is stretched. The mentioned gaps in the distribution of rates $\varrho_{q}(\gamma)$ in Eq. (4), imply that the low-frequency parts of the $\alpha$ peaks behave regularly $\chi_{q}^{\prime \prime}\left(\omega \tau^{\prime} \ll 1\right) \propto\left(\omega \tau^{\prime}\right)$. This feature is shared between $\alpha$ processes and Debye processes as one notices by comparing the dotted curves with the $\epsilon<0, n=14$ curves in Fig. 6 . The high-frequency wing of a Debye spectrum decreases linearly: $\chi_{D}^{\prime \prime}\left(\omega \tau_{D} \gg 1\right) \propto 1 /\left(\omega \tau_{D}\right)$; the $\log _{10} \chi_{D}^{\prime \prime}$ versus $\log _{10} \omega$ plot is symmetric. The $\alpha$-peak stretching is mainly caused by a sublinear decrease of the susceptibility spectrum for $1 / \tau^{\prime} \ll \omega \ll 1 / \tau_{c o}: \quad \chi_{q}^{\prime \prime}(\omega)$ $\propto 1 /\left(\omega \tau^{\prime}\right)^{b}, \quad b<1$. This also leads to the asymmetric shapes of the $\log _{10} \chi_{q}^{\prime \prime}(\omega)$ versus $\log _{10} \omega$ graphs shown in Fig. 6 for the $\alpha$ peaks.

The preceding discussion implies that the integral $\int_{0}^{\infty} \Phi(t) d t$ diverges for $\epsilon \rightarrow 0$. Therefore the Laplace transforms $\Phi_{q}(z)$ and $m_{q}(z)$ diverge for $z \rightarrow 0, \epsilon \rightarrow 0$. The Laplace transform of Eq. (3) reads $\Phi_{q}(z)=-1 /\left[z-1 /\left(i \tau_{q}\right.\right.$ $\left.\left.+m_{q}(z)\right)\right]$. In the specified limit one can neglect $\tau_{q}$ in comparison with $m_{q}(z)$, i.e., the correlators for the slow dynamics near the transition point solve the equation $\Phi_{q}(z)=-1 /\left[z-1 / m_{q}(z)\right]$, or equivalently [37]

$$
\Phi_{q}(z) /\left[1+z \Phi_{q}(z)\right]=\mathcal{L}\left[\mathcal{F}_{q}(\Phi(t))\right](z)
$$

The following calculations of this paper will deal with the analytic discussion of Eq. (19) with the aim to provide an understanding of the Figs. 4-7. The parameters $\tau_{q}$ do not occur in Eq. (19) and therefore this equation cannot define a time scale. Indeed, Eq. (19) is scale invariant: if the set of correlators $\Phi_{q}(t), q=1, \ldots, M$, solves Eq. (19), the same is true for $\Phi_{q}^{y}(t)=\Phi_{q}(y t)$ for every $y>0$. One overall time scale $t_{0}$ for the solution has to be determined by matching the $M$ correlators $\Phi_{q}(t)$ to the transient.

\section{THE TWO TIME FRACTALS}

In this section we will discuss how the appearance of two relaxation steps is related to the appearance of two fractal power laws in time, which is quantified by two anomalous exponents $a$ and $b$. The critical exponent $a$ describes the low-frequency critical susceptibility spectrum and the von Schweidler exponent $b$ quantifies the high-frequency $\alpha$-peak wings. In Sec. V A a set of auxiliary results will be derived, which is needed for the discussion of the fractal decay laws. These results will be used, in particular, to determine the range of validity of the power-law description of the critical dynamics (Sec. VB) and the range of validity of the von Schweidler-law description of the $\alpha$ process (Sec. V C).

\section{A. The two anomalous exponents}

Let us introduce a function $g_{q}(t)$ and its Laplace transform $g_{q}(z)$ so that the correlators are represented as

$$
\begin{gathered}
\Phi_{q}(t)-f_{q}^{c}=\left(1-f_{q}^{c}\right)^{2} g_{q}(t) ; \\
-z \Phi_{q}(z)-f_{q}^{c}=\left(1-f_{q}^{c}\right)^{2}\left[-z g_{q}(z)\right] .
\end{gathered}
$$

The functions $g_{q}(t)$ and $\left[-z g_{q}(z)\right]$ are generalizations of the constants $g_{q}$, which were considered in Sec. III B. Both functions reduce to these constants if the limit $1 / t \rightarrow 0$ or $z \rightarrow 0$, respectively, is considered for the glass. Both functions are used as small quantities in the following. Substituting Eq. (20a) into Eq. (19) yields the equations of motion

$$
\begin{aligned}
& z g_{q}(z) /\left[1+\left(1-f_{q}^{c}\right) z g_{q}(z)\right] \\
& \quad=\left[f_{q}^{c} /\left(1-f_{q}^{c}\right)\right]+z \mathcal{L}\left[\mathcal{F}_{q}\left(f_{k}^{c}+\left(1-f_{k}^{c}\right)^{2} g_{k}(t)\right)\right](z) .
\end{aligned}
$$

Let us specialize the preceding formulas to the critical point $\varphi=\varphi_{c}$. Expansion of Eq. (20b) leads to Eq. (10a) with $g_{k}$ replaced by $\left[-z g_{k}(z)\right]$. The inhomogeneity $I_{q}$ in this equation is also generalized to a $z$-dependent function $I_{q}(z)=I_{q}^{(1)}(z)+I_{q}^{(2)}(z)$. The first part is easily recognized as a modification of Eq. (10c)

$$
\begin{aligned}
I_{q}^{(1)}(z)= & -\left(1-f_{q}^{c}\right)\left[-z g_{q}(z)\right]^{2} \\
& -\sum_{k p} C_{q k p}^{c} z \mathcal{L}\left[g_{k}(t) g_{p}(t)\right](z) .
\end{aligned}
$$

The second part is a modification of Eq. (10d)

$$
\begin{aligned}
I_{q}^{(2)}(z)= & -\left(1-f_{q}^{c}\right)^{2}\left[-z g_{q}(z)\right]^{3} \\
& -\sum_{k p l} C_{q k p l}^{c} z \mathcal{L}\left[g_{k}(t) g_{p}(t) g_{l}(t)\right](z)+O\left(g^{4}\right) .
\end{aligned}
$$

The solution shall be constructed by an obvious generalization of the one carried out above for Eqs. (10), (12). One writes in analogy to Eq. (12b) $g_{q}(t)=g(t) e_{q}+\widetilde{g_{q}}(t)$, and notices that $\widetilde{g}_{q}(t)$ is of higher order than $g(t)$. Therefore, one gets

$$
\begin{aligned}
z \widetilde{g_{q}}(z)= & \sum_{p} R_{q p}\left\{\left(1-f_{p}^{c}\right) e_{p}^{2}[-z g(z)]^{2}\right. \\
& \left.+\sum_{k l} C_{p k l}^{c} e_{k} e_{l} z \mathcal{L}\left[g(t)^{2}\right](z)\right\}+O\left(g^{3}\right) .
\end{aligned}
$$

The expression for $I_{q}^{(1)}(z)$ is noted as a sum of second- and third-order terms in addition to unspecified contributions of fourth order 


$$
\begin{aligned}
I_{q}^{(1)}(z)= & -\left(1-f_{q}^{c}\right) e_{q}^{2}[-z g(z)]^{2} \\
& -\sum_{k p} C_{q k p}^{c} e_{k} e_{p} z \mathcal{L}\left[g(t)^{2}\right](z) \\
& -2\left(1-f_{q}^{c}\right) e_{q}\left[z^{2} g(z) \widetilde{g}_{q}(z)\right] \\
& -2 \sum_{k p} C_{q k p}^{c} e_{k} z \mathcal{L}\left[g(t) \widetilde{g}_{p}(t)\right](z)+O\left(g^{4}\right) .
\end{aligned}
$$

The expression for $I_{q}^{(2)}(z)$ starts with terms of third order

$$
\begin{aligned}
I_{q}^{(2)}(z)= & \left(1-f_{q}^{c}\right)^{2} e_{q}^{3}[z g(z)]^{3}-\sum_{k p l} C_{q k p l}^{c} e_{k} e_{p} e_{l} z \mathcal{L}\left[g(t)^{3}\right](z) \\
& +O\left(g^{4}\right) .
\end{aligned}
$$

The function $g$ in Eqs. (22) has to be evaluated from the solubility condition (12a). For a leading-order solution, only the second-order terms in Eq. (22b) have to be taken into account. Remembering the normalization condition for the eigenvectors $e_{q}, \hat{e}_{q}$ and the definition of $\lambda$ from Sec. III B, one arrives at $-z g(z)^{2}=\lambda \mathcal{L}\left[g(t)^{2}\right](z)$. This equation is solved by $g(t)=A / t^{x}$, provided the exponent $x$ obeys the equation $\Gamma(1-x)^{2} / \Gamma(1-2 x)=\lambda$. Here and in the following, $\Gamma$ denotes the $\gamma$ function. For $0<\lambda<1$ there are two solutions for the exponent $x$. One is denoted by $a$, and obeys the inequalities $0<a<1 / 2$. The other is denoted by $-b$, where $0<b$. The leading-order considerations lead to the conclusion that there are two power laws hidden in the MCT equations of motion, specified by the two anomalous exponents $a$ and $b[6,7]$. The exponents are determined by $\lambda$, which is, therefore, called the exponent parameter. For our model one gets $a=0.312, b=0.583$.

\section{B. The critical dynamics}

The critical decay is described in Eq. (20a) by a positive function $g_{q}(t)$, which decreases monotonously to zero for large $t$. Hence, the long-time critical decay can be evaluated by the methods of Sec. V A. In the preceding paragraph the solution $x=a$ has to be chosen and $A$ has to be taken positive. The constant $A$ can be written in terms of a time $t_{0}$ : $A=t_{0}^{a}$. Remembering the definition (14) of the critical amplitude, one therefore obtains as a leading-order result for the critical correlator $\Phi_{q}^{c}(t)-f_{q}^{c}=h_{q}\left(t_{0} / t\right)^{a}$ [7]. Figure 8 shows the leading-order power-law results as dashed lines. The scale $t_{0}$ for our model was found by matching the long-time asymptotes to the solution for $\left[\Phi_{q}^{c}(t)-f_{q}^{c}\right]$ for $t \sim 10^{6}$ : $t_{0}=0.425$. The leading-order result describes the correlators within $10 \%$ for times down to $t_{1}^{*}=990$ for $q_{1}$ and $t_{2}^{*}=14$ for $q_{2}$. The leading-order susceptibility spectra $\chi_{q}^{\prime \prime}(\omega)$ $=h_{q} \sin (\pi a / 2) \Gamma(1-a)\left(\omega t_{0}\right)^{a}$, shown as dashed straight lines in the double logarithmic plots of Fig. 8(b), describe the critical susceptibility spectra within a $10 \%$ accuracy level for
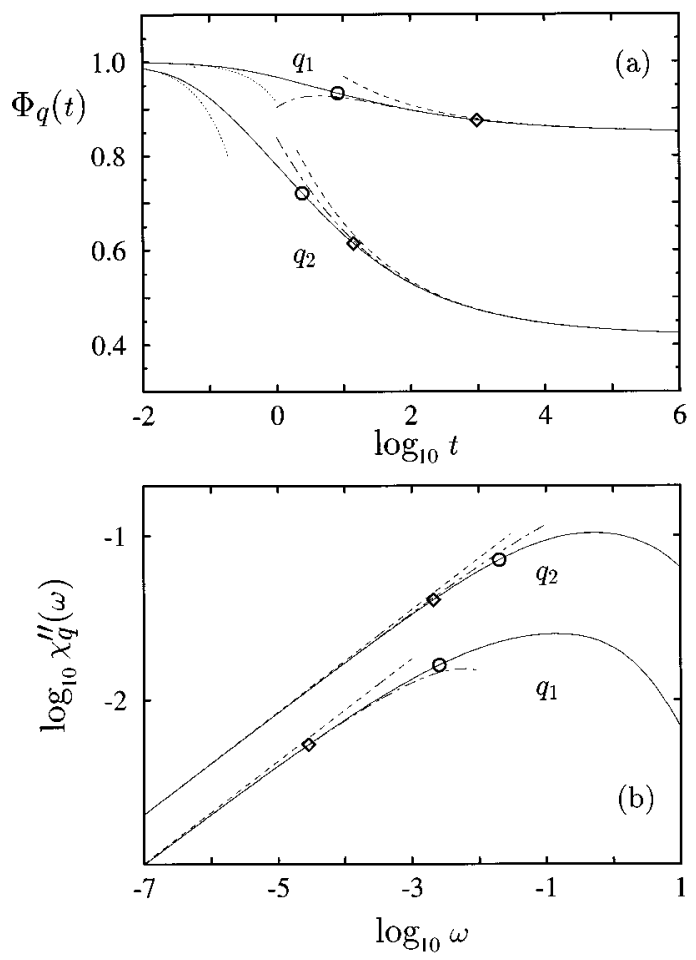

FIG. 8. The solid lines are the correlators (a) and the susceptibility spectra (b) for $q_{1}=7.0$ and $q_{2}=10.6$ at the critical point $\varphi=\varphi_{c}$. The leading-order critical laws $\Phi_{q}(t)-f_{q}^{c}=h_{q}\left(t_{0} / t\right)^{a}$ and $\chi_{q}^{\prime \prime}=\Gamma(1-a) \sin (\pi a / 2)\left(\omega t_{0}\right)^{a}, a=0.312, t_{0}=0.425$ are shown by dashes and the diamonds mark the positions $t_{q}^{*}$ and $\omega_{q}^{*}$, respectively, where they differ from the solution by $10 \%$. The dasheddotted lines present the leading-plus-next-to-leading-order results Eqs. (27). The circles mark the points where these deviate from the solutions by $10 \%$. The dotted lines in (a) show the Debye laws $\Phi_{q}^{(0)}(t)=\exp \left(-t / \tau_{q}\right)$.

frequencies below $\omega_{1}^{*}=2.9 \times 10^{-5}$ for $q_{1}$ and below $\omega_{2}^{*}=2.1 \times 10^{-3}$ for $q_{2}$. Let us recall, that the need for introducing a scale $t_{0}$ results from the scale invariance, discussed above in connection with Eq. (19).

The leading correction to the leading power law consists of two contributions. One is due to the term $\widetilde{g}_{q}(t)$ in Eq. (12b). It can be obtained by substitution of the leading approximation $g(t)=\left(t_{0} / t\right)^{a}$ into Eq. (22a). The result shall be written as $\widetilde{g}_{q}(t)=e_{q} K_{q}\left(t_{0} / t\right)^{2 a}$, so that

$$
K_{q}=\sum_{p} R_{q p}\left[\sum_{k l} C_{p k l}^{c} e_{k} e_{l}-\lambda\left(1-f_{p}^{c}\right) e_{p}^{2}\right] / e_{q} .
$$

The amplitudes $K_{q}$ for our model are shown in Fig. 2(c); in particular, one gets $K_{1}=-1.02$ and $K_{2}=-0.183$. The result for $\widetilde{g_{q}}$ can be substituted into Eq. (22b) so that $I_{q}(z)$ $=I_{q}^{(1)}(z)+I_{q}^{(2)}(z)$ is expressed in terms of $g^{2}, g^{3}$ and unspecified terms $O\left(g^{4}\right)$. The other contribution to the leading correction is found by solving Eq. (12a) with the expansion $g(t)=\left(t_{0} / t\right)^{a}+\kappa\left(t_{0} / t\right)^{2 a}+O\left(1 / t^{3 a}\right)$. One finds $\kappa=\kappa(a)$, where the function $\kappa(x)$ is determined by two constants $\xi$ and $\zeta$ 


$$
\begin{gathered}
\xi=\sum_{q} \hat{e}_{q}\left[\sum_{k p} C_{q k p}^{c} e_{k} e_{p} K_{p}+\frac{1}{2} \sum_{k p l} C_{q k p l}^{c} e_{k} e_{p} e_{l}\right], \\
\zeta=\sum_{q} \hat{e}_{q}\left[\left(1-f_{q}^{c}\right) e_{q}^{2} K_{q} \frac{1}{\lambda}+\frac{1}{2}\left(1-f_{q}^{c}\right)^{2} e_{q}^{3}\right], \\
\kappa(x)=\left[\xi \Gamma(1-3 x)-\zeta \Gamma(1-x)^{3}\right] /[\Gamma(1-x) \Gamma(1-2 x)-\lambda \Gamma(1-3 x)] .
\end{gathered}
$$

As a result one finds for the critical correlator up to errors of order $\left(t_{0} / t\right)^{3 a}$

$\Phi_{q}^{c}(t)-f_{q}^{c}=h_{q}\left(t_{0} / t\right)^{a}\left\{1+\left[K_{q}+\kappa(a)\right]\left(t_{0} / t\right)^{a}\right\}$.

This formula is equivalent to the approximation of the critical susceptibility spectra up to errors of order $\left(\omega t_{0}\right)^{3 a}$ by

$$
\begin{aligned}
\chi_{q}^{c \prime \prime}(\omega)= & h_{q} \sin (\pi a / 2) \Gamma(1-a)\left(\omega t_{0}\right)^{a}\left\{1+\left[K_{q}+\kappa(a)\right]\right. \\
& \left.\times k_{a}\left(\omega t_{0}\right)^{a}\right\}
\end{aligned}
$$

where $k_{a}=2 \cos (\pi a / 2) \Gamma(1-a) / \lambda$. For our model one obtains $\xi=0.0422, \zeta=0.269, \kappa(a)=-0.00165, k_{a}=3.16$.

Equations (27) explain the range of validity of the leading-order power laws. The corrections reach $10 \%$ for times $t_{q}^{*}$ or frequencies $\omega_{q}^{*}$, where in leading order

$$
t_{q}^{*} / t_{0}=\left[10\left(K_{q}+\kappa(a)\right)\right]^{1 / a} ; \quad \omega_{q}^{*} t_{0}=1 /\left[\left(t_{q}^{*} / t_{0}\right) k_{a}^{1 / a}\right] .
$$

As illustrated in Fig. 8(a), formula (27a) describes the critical dynamics within $10 \%$ for times down to 8.2 and 2.4 for $q_{1}$ and $q_{2}$, respectively. The result (27b) accounts for the critical spectra on a $10 \%$ accuracy level for frequencies up to $2.6 \times 10^{-3}$ and $1.9 \times 10^{-2}$ for $q_{1}$ and $q_{2}$, respectively, as is demonstrated in Fig. 8(b). Incorporating the leading corrections extends the range of validity of the analytical formulas for the critical dynamics by nearly one decade for $q_{2}$ and by about two decades for $q_{1}$. Figure 8(a) exhibits as dotted lines the Debye laws, which describe the transient dynamics in a leading-order approximation. There appears only a small window for the crossover from short-time normal liquid dynamics to the structural-relaxation dynamics.

Three features of the preceding results should be emphasized. First, the long-time or low-frequency dynamics as quantified by Eqs. (27) is determined by the mode-coupling functional $\mathcal{F}_{q}$. This holds except for the single scale $t_{0}$. This number $t_{0}$ quantifies the matching of the long-time decays for all $q$ to the transient. The transient depends on $q$ as is obvious from Figs. 4 or 6 ; and one reason for this is the strong $q$ dependence of the time scales $\tau_{q}$ Eq. (6). This $q$ dependence of the transient leads to the $q$ dependence of the size of the window where neither the short-time behavior nor Eq. (27) describe the correlators.

Second, Fig. 8 shows that the range of validity of the leading-order results depends on $q$. This range is much larger for $q_{2}$ than for $q_{1}$. The reason is the $q$ dependence of $K_{q}$ [compare Fig. 2(c)], which enters the results via Eqs. (27) or (28). One infers from Fig. 2(c), that there is one value for $q$, where the correction factor $\left[K_{q}+\kappa(a)\right]$ in Eqs. (27) al- most vanishes. In this case the range of validity of the $t^{-a}$ law is particularly large. The amplitudes $K_{q}$ also enter the following formulas (30) and their $q$ dependence will be explained in that context.

Third, the time $t_{q}^{*}$ characterizing the onset of the $1 / t^{a}$ law and the frequency $\omega_{q}^{*}$ characterizing the onset of the $\omega^{a}$ law are not related by the naive request of $\omega_{q}^{*} t_{q}^{*}$ being unity. Rather, one gets $\omega_{q}^{*} t_{q}^{*}=k_{a}^{-1 / a}$. For our model $\omega_{q}^{*}$ $=0.025 / t_{q}^{*}$. The size of the frequency window between the microscopic excitation peak, located at $\log _{10} \omega \sim 0$, and the onset $\log _{10} \omega_{q}^{*}$ of the $\omega^{a}$ spectrum is larger than the size of the corresponding time window between $\log _{10} t \sim 0$ and $\log _{10} t_{q}^{*}$. In this sense, one concludes that it is more difficult to detect the critical decay in the frequency domain than in the time domain.

\section{The von Schweidler dynamics}

In this subsection, the second time fractal shall be identified as the initial part of the second relaxation step. To proceed, we consider times on the $\alpha$-relaxation scale $\tau^{\prime}$ by writing $t=\widetilde{t} \tau^{\prime}, \Phi_{q}(t)=\widetilde{\Phi}_{q}(\widetilde{t})$. Then, we carry out the limit $\epsilon \rightarrow 0-, \tau^{\prime} \rightarrow \infty$ for fixed $\widetilde{t}$ and $\widetilde{\Phi}_{q}$. Thereby, the $\alpha$ process is separated not only from the transient but also from the first relaxation step. The function $\widetilde{\Phi}_{q}(\widetilde{t})$ describes the decay from the critical plateau, $\widetilde{\Phi}_{q}(\widetilde{t} \rightarrow 0)=f_{q}^{c}$, to zero, $\widetilde{\Phi}_{q}(\widetilde{t \rightarrow \infty})=0$. From the MCT equations of motion (1), (2), one thereby obtains the equation of motion for the $\alpha$ process [68]

$$
\begin{gathered}
\widetilde{\Phi}_{q}(\widetilde{t})=\widetilde{m}_{q}(\widetilde{t})-(d / d \widetilde{t}) \int_{0}^{\vec{t}} \widetilde{m}_{q}\left(\widetilde{t}-\widetilde{t}^{\prime}\right) \widetilde{\Phi}_{q}(\widetilde{t}) d \widetilde{t}^{\prime}, \\
\tilde{m}_{q}(\widetilde{t})=\mathcal{F}_{q}^{c}(\widetilde{\Phi}(\widetilde{t})) .
\end{gathered}
$$

This result can also be obtained from Eq. (19) if the limit $\epsilon \rightarrow 0$ is considered for the mentioned initial conditions. Again, Eq. (29) is scale invariant and does not allow to fix the time scale $\tau^{\prime}$ for the $\alpha$ process. The scale will be determined in Sec. VIC below. The general implications of Eq. (29) will also be considered below in Sec. VII.

To understand the initial part of the $\alpha$ process we write $\widetilde{\Phi}_{q}(\widetilde{t})-f_{q}^{c}=\left(1-f_{q}^{c}\right)^{2} g_{q}(\widetilde{t})$ and solve Eq. (29) by expansion in the small quantity $g_{q}(\widetilde{t})$. This leads to the same equations, which were derived in Sec. V A. Therefore, one finds in leading order $g_{q}(\widetilde{t})=e_{q} g(\widetilde{t})$ with $g(\widetilde{t})=A / \widetilde{t}^{x}$. We seek a solution which decreases with increasing $\tilde{t}$ and which vanishes for $\widetilde{t} \rightarrow 0$. Therefore, from the two possibilities for $x$ identified in the last paragraph of Sec. V A, we have to choose $x=-b$, and we must require $A=-B, B>0$. The constant 

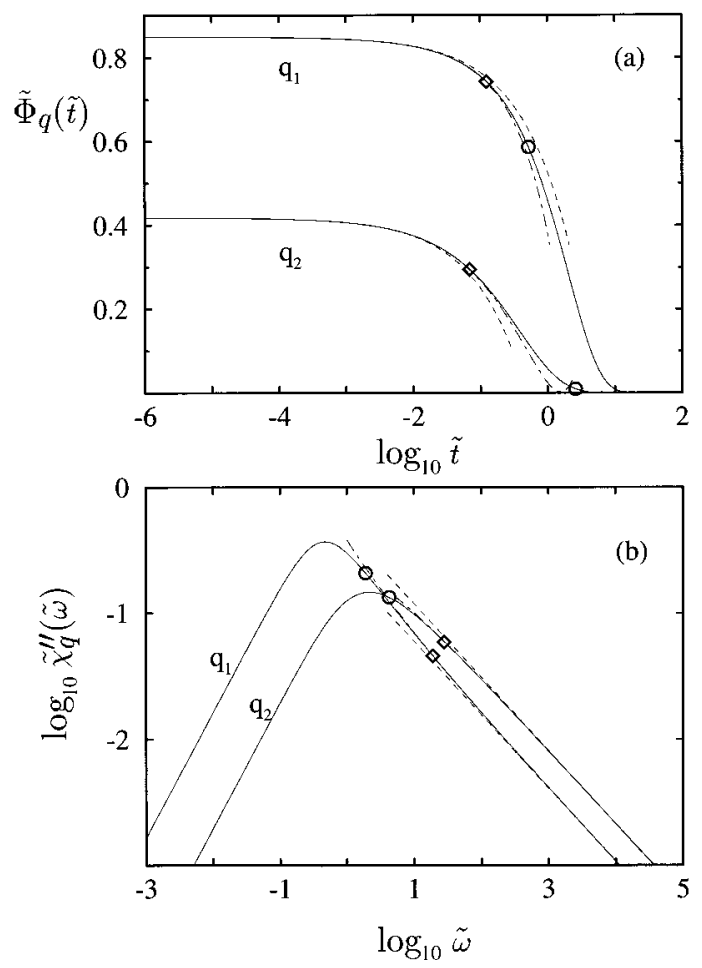

FIG. 9. The solid lines are the $\alpha$-decay correlators $\widetilde{\Phi}_{q}(\widetilde{t})$ (a) and the corresponding $\alpha$-susceptibility spectra $\widetilde{\chi}_{q}^{\prime \prime}(\widetilde{\omega})$ (b) for the two wave vectors $q_{1}=7.0$ and $q_{2}=10.6$ obtained as solution of Eq. (29). The dashed lines are the results for the von Schweidler asymptote $\widetilde{\Phi}_{q}(\widetilde{t})-f_{q}^{c}=-h_{q} \widetilde{t}^{b}, b=0.583$; they describe the solutions within $10 \%$ up to the points $\widetilde{t}_{q}^{*}$ and $\widetilde{\omega}_{q}^{*}$, respectively, marked by diamonds. The dashed-dotted lines are the leading-plus-next-toleading-order approximations, Eqs. (30). They deviate from the solutions by $10 \%$ at the points marked by circles.

$B$ can be absorbed in the time scale $\tau^{\prime}$. The scale depends on the separation parameter $\sigma$, and-with the mentioned conventions - it shall be denoted by $t_{\sigma}^{\prime}$. In leading order one, therefore, obtains $\widetilde{\Phi}_{q}(t)-f_{q}^{c}=-h_{q}\left(t / t_{\sigma}^{\prime}\right)^{b}[7,68]$. This formula is the von Schweidler law [3], mentioned in the Introduction. The leading-order correction can be obtained as explained in Sec. VB. One gets up to terms of order $\widetilde{t}^{3 b}$

$$
\widetilde{\Phi}_{q}(t)-f_{q}^{c}=-h_{q} \widetilde{t}^{b}\left\{1-\left[K_{q}+\kappa(-b)\right] \widetilde{t}^{b}\right\}, \quad \tilde{t}=t / t_{\sigma}^{\prime} .
$$

This result is equivalent to the description of the highfrequency $\alpha$-peak tail up to terms of order $1 / \widetilde{\omega}^{3 b}$ by

$$
\begin{aligned}
\widetilde{\chi}_{q}^{\prime \prime}(\omega)= & h_{q} \sin (\pi b / 2) \Gamma(1+b)\left[1 / \widetilde{\omega}^{b}\right]\left\{1-\left[K_{q}+\kappa(-b)\right]\right. \\
& \left.\times k_{b} / \widetilde{\omega}^{b}\right\}, \quad \widetilde{\omega}=\omega t_{\sigma}^{\prime} .
\end{aligned}
$$

The amplitudes $K_{q}$ and the function $\kappa(x)$ have been defined in Eqs. (23) and (26), respectively, and $k_{b}=2 \cos (\pi b / 2) \Gamma(1+b) / \lambda$. For our model one finds $\kappa(-b)=0.569$ and $k_{b}=1.48$.

The leading corrections determine the range of validity of the von Schweidler asymptotes. These describe the initial part of the $\alpha$ decay within $10 \%$ up to some value $\widetilde{t}_{q}^{*}$ for the rescaled time $\tilde{t}$. The von Schweidler law accounts for the $\alpha$-peak tails of the susceptibility spectrum within $10 \%$ down to some value $\widetilde{\omega}_{q}^{*}$ for the rescaled frequency $\widetilde{\omega}$. From Eqs. (30) one obtains in leading order

$$
\widetilde{t}_{q}^{*}=1 /\left[10\left(K_{q}+\kappa(-b)\right)\right]^{1 / b} ; \quad \widetilde{\omega}_{q}^{*}=k_{b}^{1 / b} / \widetilde{t}_{q}^{*}
$$

These results are demonstrated in Fig. 9, where the values $\widetilde{\omega}_{1}^{*}=19, \widetilde{\omega}_{2}^{*}=28$ and $\widetilde{t}_{1}^{*}=0.12, \widetilde{t}_{2}^{*}=6.8 \times 10^{-2}$ are indicated by diamonds. Within the interval $\tilde{t} \leqslant \tilde{t}_{q}^{*}$ the $\alpha$ correlator decays from $f_{q}^{c}$ to $0.88 f_{q}^{c}$ for $q_{1}$ and to $0.71 f_{q}^{c}$ for $q_{2}$. The von Schweidler law describes, on a $10 \%$ accuracy level, that part of the $\alpha$ spectrum, where $\chi_{q}^{\prime \prime}(\omega) / \chi_{\max }(q)$ is smaller than 0.12 for $q_{1}$ and smaller than 0.40 for $q_{2}$. In the same sense as discussed in the last paragraph of Sec. V B, it is more difficult to identify the von Schweidler fractal in the frequency domain than in the time domain, because $\widetilde{\omega}_{q}^{*} \widetilde{t}_{q}^{*}=k_{b}^{1 / b}$ is larger than unity. For our model $\widetilde{\omega}_{q}^{*}=2.0 / \widetilde{t}_{q}^{*}$.

The leading corrections extend the window of validity of the analytic description by about an order of magnitude, as can be inferred from Fig. 9. The points where the results, Eqs. (30), deviate from the full solutions by $10 \%$ are marked by circles in the figures. On the specified $10 \%$ accuracy level, Eq. (30a) describes the decay of the $\alpha$ correlator down to $69 \%$ of its initial value for $q_{1}$ and down to $2.3 \%$ for $q_{2}$. For $q=q_{2}$, the correction factor $-\left[K_{q}+\kappa(-b)\right]$ in Eq. (30) is negative. Therefore, the high-frequency part of the $\alpha$ peak is a convex curve in the $\log _{10} \chi^{\prime \prime}$ versus $\log _{10} \omega$ plot of Fig. 9(b). According to Fig. 2(c) the correction factor is larger for $q_{1}$ than for $q_{2}$, indeed, it is positive. Therefore, the highfrequency $\alpha$-peak wing in Fig. 9(b) is, concave for $q_{1}$. The upper part of the $\alpha$ peak is, therefore, narrower for $q_{1}$ than for $q_{2}$. The half width at half maximum height is 1.64 decades for $q_{2}$; i.e., this peak is 3.1 times broader than a Debye peak. The peak for $q_{1}$ has a half width of 1.30 decades, i.e., it is only 1.4 times broader than a Debye spectrum (compare in Fig. 6 the dotted lines with the $\epsilon=-10^{-14 / 3}$ results). The wave-vector dependence of $K_{q}$ causes the stretching to be more pronounced for the wave vector $q_{2}$ near the structurefactor-minimum position than for the wave vector $q_{1}$ near the structure-factor-peak position. There is a wave vector between $q_{1}$ and $q_{2}$ and another one between $q_{0}$ and $q_{1}$, where the correction factor almost vanishes. For these two values of $q$ the range of validity of von Schweidler's law is particularly large.

The essential wave vectors, which drive the liquid-glass transition, are located within a shell around the structurefactor-peak position, say, $6 \leqq q d \leqslant 14$. It was explained in Sec. III, why in this shell $f_{q}^{c}$ oscillates in phase with $S_{q}$, and why $h_{q}$ oscillates opposite in phase; in particular, $f_{1}^{c}>f_{2}^{c}$ and $h_{1}<h_{2}$ [compare Fig. 2(b)]. The equations of motion (29) couple the correlators strongly within the mentioned shell. Therefore, the correlators have a tendency to reach zero roughly at the same time. At the structure-factor-peak position $\widetilde{\Phi}_{q}(\widetilde{t})$ has to achieve a larger decay than away from this position; and this has to happen even though the amplitude $h_{q}$ for the initial decay is smaller than the one near the structure-factor-minimum position. Consequently, at the structure-factor peak the smallest stretching results. For the decay of $\Phi_{1}$ to catch up with that of $\Phi_{2}$ it is necessary that 
the correction factor $-\left[K_{q}+\kappa(-b)\right]$ in Eq. (30a) is larger for $q_{1}$ than for $q_{2}$, i.e., $K_{1}<K_{2}$. This explains the pronounced minimum of the $K_{q}$ versus $q$ graph for $q \sim 7$ in Fig. 2(c), and therefore the wave-vector dependence of the $\alpha$-process stretching. It is the same amplitude $K_{q}$ which quantifies the deviation of the critical law from the leadingorder asymptote in Eqs. (27). Thus the $q$ dependence of the latter result is understood qualitatively as well.

\section{THE FIRST SCALING-LAW REGIME}

The regularity properties of the MCT solutions imply that in the vicinity of the glass transition there is a time window where the correlators $\Phi_{q}(t)$ are close to the critical plateau $f_{q}^{c}$. In the frequency domain, this condition implies the existence of a window where the susceptibility $\chi_{q}(\omega)$ is close to $1-f_{q}^{c}$

$$
\left|\Phi_{q}(t)-f_{q}^{c}\right| \ll 1, \quad\left|\chi_{q}(\omega)-\left(1-f_{q}^{c}\right)\right| \ll 1 .
$$

If $\epsilon=\left(\varphi-\varphi_{c}\right) / \varphi_{c}$ decreases to zero, the length of this time window diverges. Within the short-time part of this window, the correlators decay towards $f_{q}^{c}$. Within the glass, and $t$ near $\tau_{c o}=t_{q}^{+}$, the dynamics deals with the crossover to arrest at the long-time limit $f_{q}$. For the liquid, the correlators cross the plateau at $\tau_{c o}=t_{q}^{-}$and then approach the von Schweidler decay for longer times. The dynamics within the specified windows is called MCT $\beta$ relaxation [37]. In Sec. VIA, it will be demonstrated that the equations of motion can be simplified considerably for the $\beta$-relaxation process. In Sec. VIB. it will be discussed how the leading-order terms yield a scaling-law description of the $\beta$ dynamics. The range of validity of the scaling-law description is discussed in Sec. VIC.

\section{A. The equations of motion for the relaxation near the critical plateau $f_{q}^{c}$}

Let us start with the equations of motion (20b). The conditions (32) are then equivalent to the statement that-in addition to $\epsilon-g_{q}(t)$ and $z g_{q}(z)$ can be treated as small quantities. The following calculation therefore is a combination of the expansion procedures carried out in Secs. IIIB, IIIC, and V A. One arrives at the analog of Eq. (10a)

$$
\sum_{k}\left[\delta_{q k}-C_{q k}^{c}\right]\left[-z g_{k}(z)\right]=J_{q}(z)
$$

In analogy to Eq. (10b), the inhomogeneity consists of two contributions: $J_{q}(z)=J_{q}^{(1)}(z)+J_{q}^{(2)}(z)$. These combine the results of Eqs. (10c), (21a) and (10d), (21b), respectively

$$
\begin{gathered}
J_{q}^{(1)}(z)=\Delta C_{q}+I_{q}^{(1)}(z), \\
J_{q}^{(2)}(z)=\sum_{k} \Delta C_{q k}\left[-z g_{k}(z)\right]+I_{q}^{(2)}(z) .
\end{gathered}
$$

The solution of Eq. (33a) can be written in the form of Eq. (12b): $g_{q}(z)=g(z) e_{q}+\widetilde{g}_{q}(z)$ or $g_{q}(t)=g(t) e_{q}+\widetilde{g}_{q}(t)$. As before, one shows that the parts $\vec{g}_{q}$ only contribute to the next-to-leading terms. Therefore, they can easily be evalu- ated and are found to consist of terms proportional to the amplitude $\bar{K}_{q}$ from Eq. (16) and other terms proportional to the amplitude $K_{q}$ from Eq. (23). It is more cumbersome to determine $g(z)$ or $g(t)$ from the solubility condition (12a). Via Eq. (14) this term leads to a contribution proportional to the critical amplitude $h_{q}$. Let us write $g(t)$ as a sum of a leading-order contribution $G(t)$ and a leading correction: $g(t)=G(t)+[H(t)+\sigma \times$ const $]$. The equation of motion for $G(t)$ is obtained by working out the leading approximation of Eq. (12a): $\Sigma_{q} \hat{e}_{q} J_{q}^{(1)}(z)=0$. Remembering the definitions of the separation parameter $\sigma$ and of the exponent parameter $\lambda$ from Sec. IIIB one gets $[6,7]$

$$
\sigma-[z G(z)]^{2}=\lambda z \mathcal{L}\left[G(t)^{2}\right](z) .
$$

This result can be used to eliminate Laplace transforms of $G(t)^{2}$ in terms of $G(z)^{2}$ and vice versa. One obtains

$$
\begin{aligned}
-z \widetilde{g}_{q}(z) & =K_{q} e_{q} \frac{1}{\lambda}\left[z^{2} G(z)^{2}-\frac{\sigma}{1-\lambda}\right]+\bar{K}_{q} e_{q} \frac{\sigma}{\sqrt{1-\lambda}}, \\
\widetilde{g}_{q}(t) & =K_{q} e_{q}\left[G(t)^{2}-\frac{\sigma}{1-\lambda}\right]+\bar{K}_{q} e_{q} \frac{\sigma}{\sqrt{1-\lambda}},
\end{aligned}
$$

as well as the equation of motion for $H$

$$
\begin{aligned}
\lambda \mathcal{L} & {[G(t) H(t)](z)+z G(z) H(z) } \\
\quad & =-\xi \mathcal{L}\left[G(t)^{3}\right](z)-\lambda \zeta z G(z) \mathcal{L}\left[G(t)^{2}\right](z) .
\end{aligned}
$$

The numbers $\xi$ and $\zeta$ have been introduced above in Eqs. (24) and (25). An equation equivalent to Eq. (35c) has been derived before [8] for MCT models dealing with the case $M=1$. The general case extends the $M=1$ one in the definition of the two numbers $\xi$ and $\zeta$. The appearance of the terms proportional to $\bar{K}_{q}$ and $K_{q}$ has no analogy within $M=1$ models.

Combining the preceding results one finds for the correlators in leading-plus-next-to-leading order

$$
\Phi_{q}(t)-f_{q}^{c}=h_{q}\left\{G(t)+\left[H(t)+K_{q} G(t)^{2}+\sigma \overline{\bar{K}}_{q}\right]\right\} .
$$

This equation is equivalent to the formula for the dynamical susceptibility

$$
\begin{aligned}
\chi_{q}(\omega)-\left(1-f_{q}^{c}\right) \\
\quad=h_{q}\left\{z G(z)+\left[z H(z)+K_{q}\left(\sigma-z^{2} G(z)^{2}\right) / \lambda-\sigma \overline{\bar{K}}_{q}\right]\right\} .
\end{aligned}
$$

Above, the new amplitude $\overline{\bar{K}}_{q}$ abbreviates

$$
\overline{\bar{K}}_{q}=\left[\bar{K}_{q}-K_{q} / \sqrt{1-\lambda}+\kappa+(\lambda \zeta-\xi) /(1-\lambda)^{3 / 2}\right] / \sqrt{1-\lambda} .
$$

For our model one obtains: $\overline{\bar{K}}_{1}=3.57, \overline{\bar{K}}_{2}=3.78$.

\section{B. The leading-order results}

If one restricts the results (36) to the leading contributions, one obtains the factorization theorem [7] 

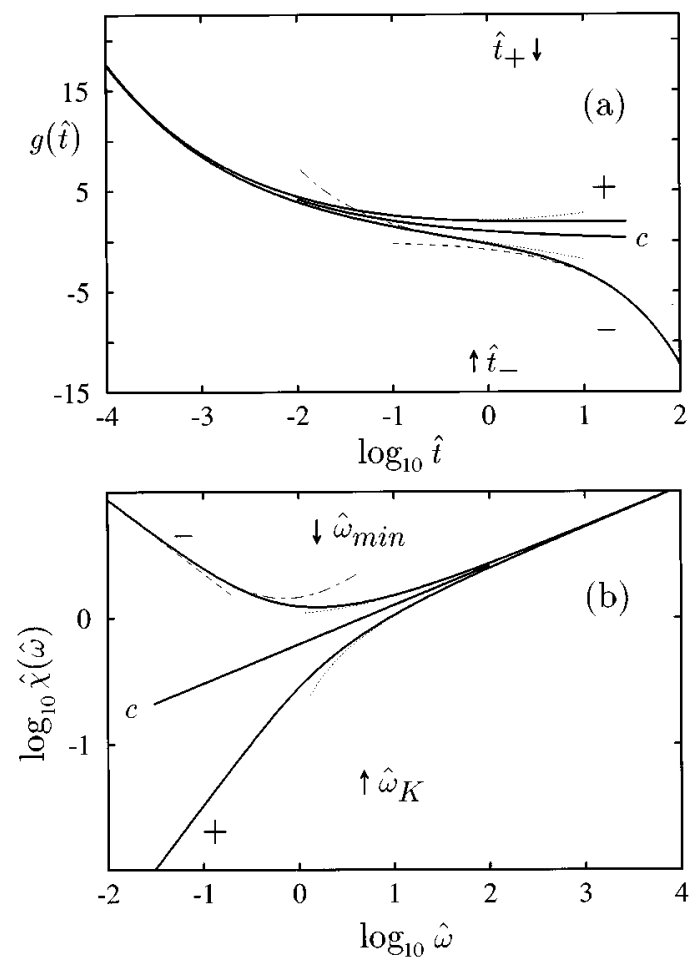

FIG. 10. (a) Correlators $1 / \hat{t}^{a}$ (curve $c$ ) and $\beta$-relaxation master functions $g_{ \pm}(\hat{t})$ (curves \pm ) for the exponent parameter $\lambda=0.735, a=0.312$. The dotted lines represent the short-time expansions $g_{ \pm}=1 / \hat{t}^{a} \pm 1.12 \hat{t}^{a}$. The dashed line exhibits the von Schweidler law $g_{-}(\hat{t})=-B \hat{t}^{b}$ and the dashed-dotted curve is the expansion $g_{-}(\hat{t})=-B \hat{t}^{b}+B_{1} /\left(B \hat{t}^{b}\right) \quad\left(b=0.583, B=0.836, \quad B_{1}=\right.$ 0.431). The arrows mark the crossover times $\hat{t}_{ \pm}$. (b) The susceptibility spectra $\hat{\chi}(\hat{\omega})$ for the results from (a). The arrows mark the minimum and knee positions.

$$
\Phi_{q}(t)-f_{q}^{c}=h_{q} G(t), \quad \chi_{q}^{\prime \prime}(\omega)=h_{q} \chi^{\prime \prime}(\omega)
$$

The deviations $\Phi_{q}(t)-f_{q}^{c}$ of the correlators from the plateau $f_{q}^{c}$ consist of two factors. The $q$ dependence is given by the critical amplitude $h_{q}$. The dependence on time and on the control parameters is described by the second factor $G(t)$. Equivalently, the spectrum $\chi_{q}^{\prime \prime}(\omega)$ consists of the first factor $h_{q}$ and of a second factor $\chi^{\prime \prime}(\omega)$ which is the absorptive part of $z G(z)$, for $z=\omega+\mathrm{i} 0$. The factorization theorem (37) reflects the center-manifold theorem of bifurcation theory [69]. One can consider $G$ as a function of the two variables $\left(t / t_{0}\right)$ and $\sigma$. This function is to be evaluated for every $\lambda$ : $G(t)=g^{\lambda}\left(t / t_{0}, \sigma\right)$. The function $g$ has been discussed comprehensively in the earlier literature [36,70], and therefore we shall quote-without proof-only those properties which are necessary to understand the following figures.

At the critical point, Eq. (34) yields the leading-order result for the critical decay from Sec. V B

$$
\begin{aligned}
G(t)= & \left(t_{0} / t\right)^{a}, \\
& \chi^{\prime \prime}(\omega)=\sin (\pi a / 2) \Gamma(1-a)\left(\omega t_{0}\right)^{a} ; \quad \sigma=0 .
\end{aligned}
$$

For nonvanishing separations, one obtains scaling laws

$$
G(t)=c_{\sigma} g_{ \pm}\left(t / t_{\sigma}\right), \quad \chi^{\prime \prime}(\omega)=c_{\sigma} \hat{\chi}_{ \pm}\left(\omega t_{\sigma}\right) ; \quad \sigma \gtrless 0 .
$$

For the correlation scale $c_{\sigma}$ and time scale $t_{\sigma}$ the following power laws are valid:

$$
c_{\sigma}=\sqrt{|\sigma|} ; \quad t_{\sigma}=t_{0} /|\sigma|^{\delta}, \quad \delta=\frac{1}{2 a}
$$

The control-parameter-independent master functions $g_{ \pm}(\hat{t})$ are obtained by solving Eq. (34) for $\sigma= \pm 1 . \hat{\chi}_{ \pm}(\hat{\omega}) / \hat{\omega}$ is the Fourier cosine transform of $g_{ \pm}(\hat{t})$. These functions depend smoothly on $\lambda$. Figure 10 shows the results for our model. The dependence of $G(t)$ or $\chi^{\prime \prime}(\omega)$ on the control parameters enters via the two scales $c_{\sigma}$ and $t_{\sigma}$. The square-root result for the correlation scale $c_{\sigma}$ is the fingerprint of the underlying fold bifurcation. The exponent $\delta$ for the time scale is also fixed by $\lambda$; depending on the model, $\delta$ can have any value larger than unity. For our model we obtain: $\delta=1.60$.

For small rescaled times $\hat{t}=t / t_{\sigma}$, the master functions approach the critical asymptote

$$
\begin{gathered}
g_{ \pm}(\hat{t})=1 / \hat{t}^{a} \pm A_{1} \hat{t}^{a}+O\left(\hat{t}^{3 a}\right), \\
A_{1}=\frac{1}{2} /[\Gamma(1+a) \Gamma(1-a)-\lambda] .
\end{gathered}
$$

For our model, one finds $A_{1}=1.12$. These formulas explain the symmetric approach of the curves \pm towards curve $c$ for $\hat{t} \leqslant 1$ in Fig. 10(a). The corresponding approach of the susceptibility spectra \pm towards the straight line $c$ in Fig. 10(b) for rescaled frequencies $\hat{\omega}=\omega t_{\sigma}>10$ is explained by $\hat{\omega}$ times the Fourier cosine transform of Eq. (41).

The glass correlator $g_{+}$approaches its long-time limit $1 / \sqrt{1-\lambda}$ exponentially [49]. A characteristic crossover time $\hat{t}_{+}$can be defined, for example, as one where the monotonically decreasing correlator reaches its long-time limit within $0.1 \%: g_{+}\left(\hat{t}_{+}\right)=1.001 / \sqrt{1-\lambda}$. One obtains a regular lowfrequency spectrum: $\hat{\chi}_{+}(\hat{\omega})=C_{0} \hat{\omega}+O\left(\hat{\omega}^{3}\right)$. The crossover from the linear susceptibility spectrum for small $\hat{\omega}$ to the sublinear critical spectrum for large $\hat{\omega}$ causes the knee of the $\log _{10} \hat{\chi}_{+}$versus $\log _{10} \hat{\omega}$ graph in Fig. 10(b). The knee frequency $\hat{\omega}_{K}$ can be defined, for example, as the position of the maximum of the $\hat{\chi}_{+}(\hat{\omega}) / \sqrt{\hat{\omega}}$ versus $\hat{\omega}$ graph. Let us denote the spectral intensity for the knee by $\hat{\chi}_{K}=\hat{\chi}_{+}\left(\hat{\omega}_{K}\right)$. For $\lambda=$ 0.735 one obtains: $\hat{t}_{+}=3.33, \hat{\omega}_{K}=4.80, C_{0}=0.326, \hat{\chi}_{K}=0.75$.

For large $\hat{t}$, the liquid correlator approaches the von Schweidler law

$$
\begin{gathered}
g_{-}(\hat{t} \gg 1)=-\left(B \hat{t}^{b}\right)+B_{1} /\left(B \hat{t}^{b}\right)+O\left(1 / \hat{t}^{3 b}\right), \\
B_{1}=\frac{1}{2} /[\Gamma(1-b) \Gamma(1+b)-\lambda] .
\end{gathered}
$$

For our model, one finds $B_{1}=0.431$. A crossover time $\hat{t}_{-}$can be defined, for example, by the zero of the decay function: $g_{-}\left(\hat{t}_{-}\right)=0$. The crossover from the von Schweidler susceptibility for low $\hat{\omega}, \hat{\chi}_{-}(\hat{\omega} \ll 1)=\sin (\pi b / 2) \Gamma(1$ $+b) B / \hat{\omega}^{b}+O\left(\hat{\omega}^{b}\right)$, to the critical susceptibility spectrum for 


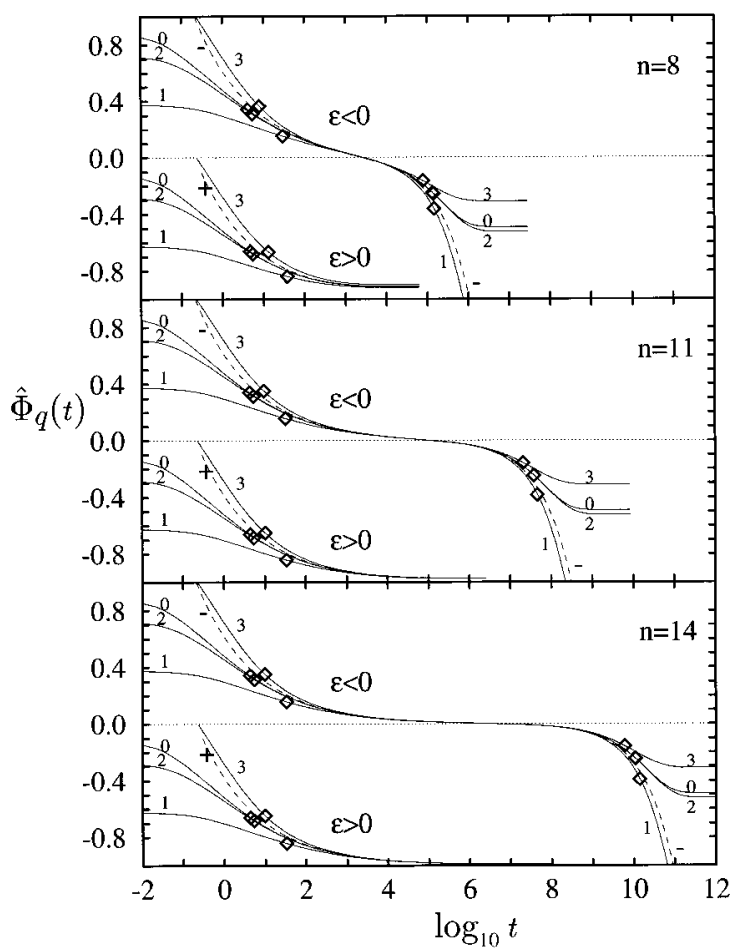

FIG. 11. Functions $\hat{\Phi}_{q}(t)=\left(\Phi_{q}(t)-f_{q}^{c}\right) /\left(h_{q} \sqrt{C}\right)$ for $q_{0}=3.4$, $q_{1}=7.0, q_{2}=10.6$, and $q_{3}=17.4$ for $\epsilon= \pm 10^{-n / 3}$ for three values of $n$ (solid lines with labels 0 to 3 ). The diamonds mark the positions where the $\hat{\Phi}_{q}(t)$ deviate by \pm 0.05 from the scaling asymptotes $G(t) / \sqrt{C}$, which are shown as dashed curves. $C=1.54$ and the curves for $\epsilon>0$ are shifted downwards by 1 , for clarity.

high $\hat{\omega}$ causes the minimum of the spectrum $\hat{\chi}_{-}$in Fig. 10(b). Let us denote the minimum position by $\hat{\omega}_{\min }$ and the minimum intensity by $\hat{\chi}_{\text {min }}=\hat{\chi}_{-}\left(\hat{\omega}_{\text {min }}\right)$. For $\lambda=0.735$ one gets: $\hat{t}_{-}=0.704, \hat{\omega}_{\text {min }}=1.56, B=0.836, \hat{\chi}_{\text {min }}=1.22$.

The asymptotic results (37), (39) - in particular, the evolution of their ranges of validity with changes of the reduced packing fraction $\epsilon$-are demonstrated in Figs. 11 and 12. In addition to the correlators for the wave vector $q_{1}$ and $q_{2}$, the small wave vector $q_{0}$, and the large wave vector $q_{3}$ are considered [compare Fig. 2(a)]. Figure 11 shows that the functions $\left(\Phi_{q}(t)-f_{q}^{c}\right) /\left(h_{q} \sqrt{C}\right)=\hat{\Phi}_{q}(t)$ agree with the scalinglaw result $G(t) / \sqrt{C}=c_{\sigma} g_{ \pm}\left(t / t_{\sigma}\right) / \sqrt{C}$ for $n=14$ within \pm 0.05 for the eight decades interval $1.5 \$ \log _{10} t \leqslant 9.5[C$ is the coefficient defined in Eq. (13)]. Similarly, Fig. 12 shows that for $n=14$ the functions $\hat{\chi}_{q}(\omega)=\chi_{q}^{\prime \prime}(\omega) /\left(h_{q} \sqrt{C}\right)$ agree with $\chi^{\prime \prime}(\omega) /(\sqrt{C})=c_{\sigma} \hat{\chi}_{ \pm}\left(\omega t_{\sigma}\right) / \sqrt{C}$ within $10 \%$ for the four decade interval $-8.5 \leqq \log _{10} \omega \lesssim-4.5$. Notice that the rescaling of the master spectrum is achieved in the double logarithmic representation of Fig. 12 by a mere translation of the plot without change of shape: the dashed lines are obtained by translating the graphs from Fig. 10 (b) by $\log _{10}\left(c_{\sigma} / \sqrt{C}\right)$ parallel to the vertical axis and by $\log _{10} t_{\sigma}$ parallel to the horizontal one. If the separation parameter is increased by a factor 10, the ranges of validity of Eqs. (37), (39) shrink by more than a factor 100 as is shown for the $n=11$ results in Figs. 11 and 12. A further increase of the separation by an-

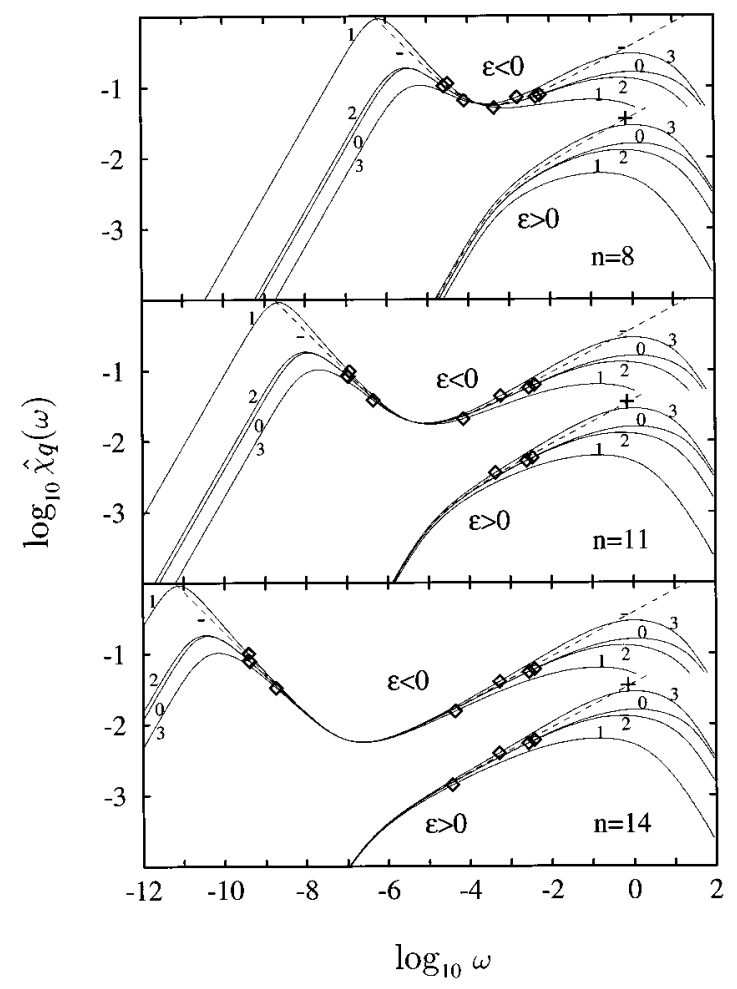

FIG. 12. The spectra $\hat{\chi}_{q}(\omega)=\chi_{q}^{\prime \prime}(\omega) /\left(h_{q} \sqrt{C}\right)$ for the results from Fig. 11 (solid lines). The diamonds mark the points where the $\hat{\chi}_{q}(\omega)$ deviate by $10 \%$ from the scaling-law asymptotes $\hat{\chi}(\omega) / \sqrt{C}$, which are shown as dashed curves. The curves for $\epsilon>0$ are shifted downwards by one decade, for clarity.

other factor 10 is analyzed for the $n=8$ results in those figures. The asymptotic laws now hold for a 3.5 decade time window, i.e., for $1.5 \leqq \log _{10} t \leqslant 5.0$ within \pm 0.05 . In the frequency domain, a similar shrinking of the window for the applicability is demonstrated. For the two wave vectors $q_{1}$ and $q_{3}$, the susceptibility spectra of the liquid deviate already qualitatively from the master spectrum $\hat{\chi}_{-}$. For the $n=8$ results, the glass spectra deviate for all wave vectors and frequencies from the master spectrum by more than $10 \%$.

In connection with Fig. 7(a), characteristic times $\tau_{q}^{ \pm}$have been defined, which quantify the crossover times $\tau_{c o}$ from the first to the second relaxation step. Corresponding times $\hat{t}_{ \pm}$have been defined above for the correlators $g_{ \pm}(\hat{t})$. Within the range of validity of the leading-order results one obtains from the scaling laws Eq. (39), the formula

$$
\tau_{q}^{ \pm}=\hat{t}_{ \pm} t_{\sigma}
$$

It is the factorization theorem which explains the $q$ independence of the scales $\tau_{q}^{ \pm}$in the limit $\sigma \rightarrow 0$, which was demonstrated in Fig. 7(c). The strong increase of the scales, which is shown in Fig. 7(a) for $|\sigma| \rightarrow 0$, is a consequence of the power-law divergence obtained for $t_{\sigma}$ in Eq. (40). The lines through the diamonds in Fig. 7(a) exhibit the result (43a) for our model. Equivalent results are obtained if one considers the positions $\omega_{\min }(q)$ and $\omega_{K}(q)$ of the susceptibility minima and knees. Again, one finds these quantities to 
$\varepsilon$

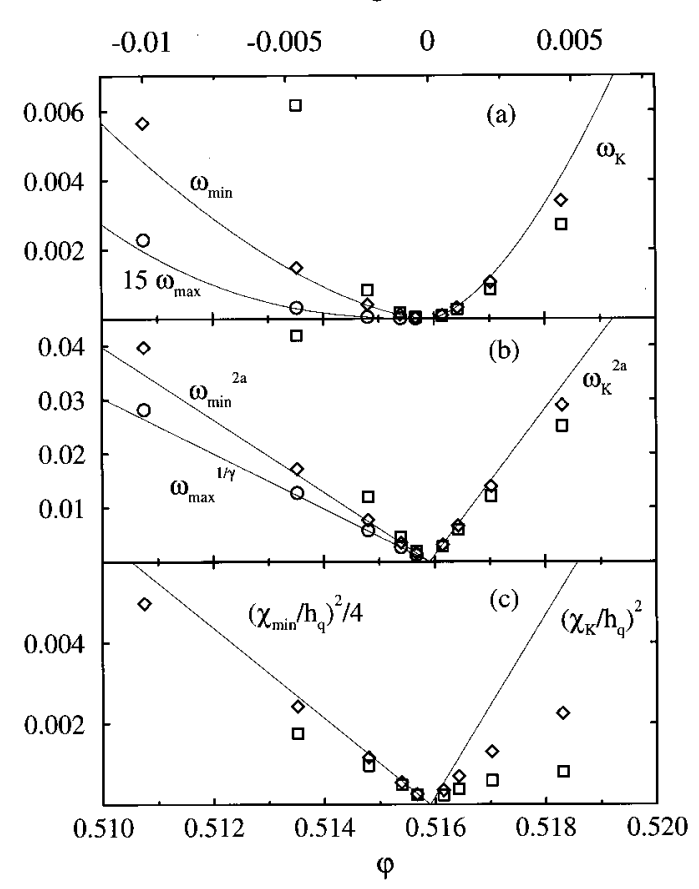

FIG. 13. (a) Positions of the susceptibility minima $\omega_{\min }(q)$ and knees $\omega_{K}(q)$ for $\varphi<\varphi_{c}$ and $\varphi>\varphi_{c}$, respectively. The squares refer to $q_{1}$ and the diamonds to $q_{2}$. The solid lines are the scaling-law asymptotes $\left[\hat{\omega}_{\min } / t_{0}\right]|\sigma|^{\delta},\left[\hat{\omega}_{K} / t_{0}\right]|\sigma|^{\delta}, \delta=1.60$. The circles are the susceptibility maxima positions $\omega_{\max }\left(q_{2}\right)$, and the line shows the power-law asymptote $\omega_{\max }=3.74|\sigma|^{\gamma} ; \gamma=2.46$. (b) $\omega_{\text {min }}^{2 a}$ and $\omega_{K}^{2 a}$ for the data from (a) in comparison with the leading-order results, Eqs. (44) (straight lines); $a=0.312$. The circles are $\omega_{\max }^{1 / \gamma}$ for the data from (a) together with the straight line asymptote. (c) $\left[\chi_{q}^{\prime \prime}\left(\omega_{\min }\right) /\left(2 h_{q}\right)\right]^{2}$ and $\left[\chi_{q}^{\prime \prime}\left(\omega_{K}\right) / h_{q}\right]^{2}$ as function of the packing fraction for the data from Fig. 6 . The straight lines are the scalinglaw asymptotes from Eqs. (45); squares refer to $q_{1}$, and diamonds to $q_{2}$.

be $q$ independent within the range of validity of the factorization theorem as is shown in Fig. 13(a). Compressing the liquid towards the critical packing fraction $\varphi_{c}$ manifests itself by an approach of $\omega_{\min }(q)$ to zero. Similarly, the melting of the glass upon expansion is reflected by a vanishing of $\omega_{K}(q)$. The scaling laws (39) yield the power-law results

$$
\omega_{\min }(q)=\hat{\omega}_{\min } / t_{\sigma}, \quad \omega_{K}(q)=\hat{\omega}_{K} / t_{\sigma}
$$

The asymptotic laws are shown as lines in Fig. 13(a). The power-law singularities for the frequency scales are more explicit in a rectification diagram, i.e., in a $\omega^{2 a}$ versus $\varphi$ representation. Within the range of validity of the leading asymptotic formulas, one obtains a linear variation with the reduced packing fraction

$$
\begin{gathered}
\omega_{\text {min }}(q)^{2 a}=\left[\hat{\omega}_{\text {min }} / t_{0}\right]^{2 a} C\left(\varphi_{c}-\varphi\right) / \varphi_{c}, \quad \varphi<\varphi_{c}, \\
\omega_{K}(q)^{2 a}=\left[\hat{\omega}_{K} / t_{0}\right]^{2 a} C\left(\varphi-\varphi_{c}\right) / \varphi_{c}, \quad \varphi>\varphi_{c} .
\end{gathered}
$$
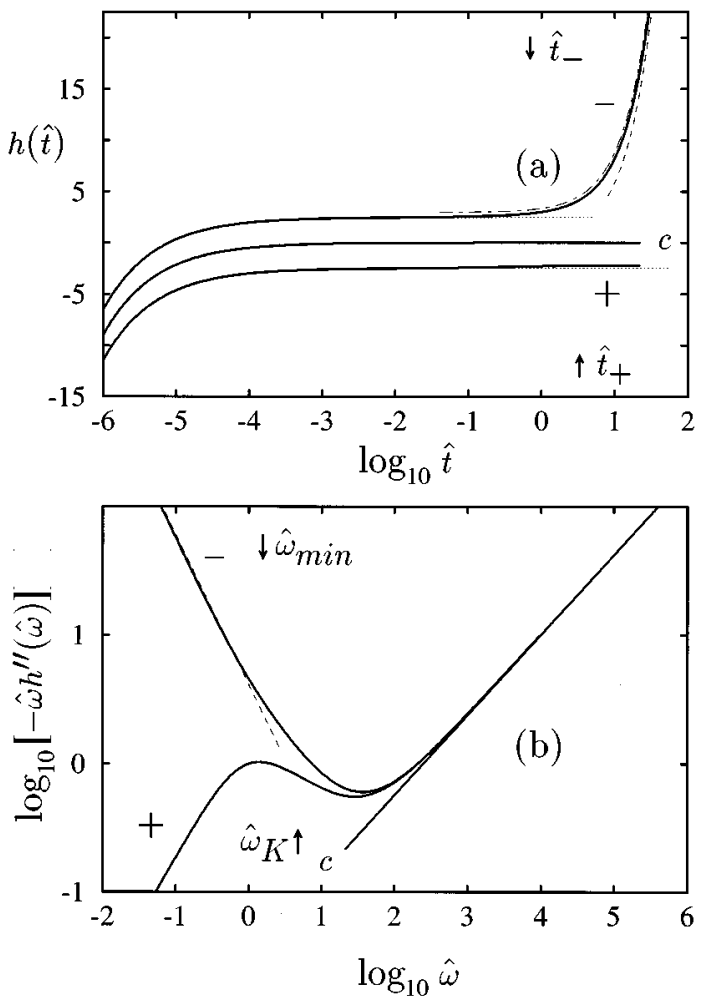

FIG. 14. Master functions $h(\hat{t})$ as function of the rescaled time $\hat{t}=t / t_{\sigma}$ (a) and the master susceptibility spectra $\hat{\omega} h^{\prime \prime}(\hat{\omega})$ as function of the rescaled frequency $\hat{\omega}=\omega t_{\sigma}$ (b) for the corrections to the $\beta$-relaxation scaling laws for $\lambda=0.735, \xi=0.0422, \quad \zeta=0.269$. Curves $c$ refer to the critical point and curves \pm to glass and liquid, respectively. The dotted lines in (a) exhibit the asymptotic laws $h_{ \pm}=-0.00165 / \hat{t}^{2 a} \mp 2.48$. The dashed-dotted line is the approximation $h_{-}(\hat{t})=\kappa(-b)\left(B \hat{t}^{b}\right)^{2}+\widetilde{\kappa}(-b)$, where $\kappa(-b)=0.569$, $\widetilde{\kappa}(-b)=2.97, B=0.836$. The dashed lines represent the asymptote $h_{-}(\hat{t})=\kappa(-b)\left(B \hat{t}^{b}\right)^{2}$. The arrows are the markers from Fig. 10.

The results for $\omega_{\min }(q)^{2 a}$ and $\omega_{K}(q)^{2 a}$ are compared with the asymptotic straight lines in Fig. 13(b).

The fold bifurcation also manifests itself by a vanishing of the spectral intensities at the minimum and knee proportional to the universal square-root law

$$
\chi_{\min }(q)=h_{q} \hat{\chi}_{\min } c_{\sigma} ; \quad \chi_{K}(q)=h_{q} \hat{\chi}_{K} c_{\sigma}
$$

Again, the singularity is exhibited more transparently by considering a rectification diagram, i.e., a plot of the squares of the intensities

$$
\begin{gathered}
{\left[\chi_{\min }(q) / h_{q}\right]^{2}=\left[\hat{\chi}_{\min }\right]^{2} C\left(\varphi_{c}-\varphi\right) / \varphi_{c}, \quad \varphi<\varphi_{c},} \\
{\left[\chi_{K}(q) / h_{q}\right]^{2}=\left[\hat{\chi}_{K}\right]^{2} C\left(\varphi-\varphi_{c}\right) / \varphi_{c}, \quad \varphi>\varphi_{c} .}
\end{gathered}
$$

In Fig. 13(c), the results for $\left(\chi_{\min } / h_{q}\right)^{2}$ and $\left(\chi_{K} / h_{q}\right)^{2}$ are compared to the asymptotic straight lines, showing the righthand side of Eqs. (45). 


\section{The leading corrections to the scaling laws}

The range of validity of the scaling-law description of the dynamics is $q$ dependent, as is obvious from Figs. 11-13. The $q$ dependence is determined by $\sigma \bar{K}_{q}$ and the terms proportional to $K_{q}$ in Eqs. (36). Since $G(t)^{2}=c_{\sigma}^{2} g_{ \pm}\left(t / t_{\sigma}\right)^{2}$, the $G^{2}$ terms are also given by scaling laws; the time scale is the same $t_{\sigma}$ as discussed above, but the amplitude scale is reduced from $c_{\sigma}$ to $c_{\sigma}^{2}=|\sigma|$. With $H(t)$ a new function enters. Its equation of motion can be noted in a convenient form by Laplace backtransformation of Eq. (35c)

$$
\begin{aligned}
& \lambda G(t) H(t)-(d / d t) \int_{0}^{t} G\left(t-t^{\prime}\right) H\left(t^{\prime}\right) d t^{\prime} \\
& \quad=\lambda \zeta(d / d t) \int_{0}^{t} G\left(t-t^{\prime}\right) G\left(t^{\prime}\right)^{2} d t^{\prime}-\xi G(t)^{3} .
\end{aligned}
$$

The solution for $\varphi=\varphi_{c}$, which reproduces via Eq. (36a) the results of Sec. VB for the critical relaxation, reads

$$
H(t)=\kappa(a)\left(t_{0} / t\right)^{2 a} ; \quad \sigma=0
$$

Here, $\kappa(x)$ was defined in Eq. (26). For nonvanishing separations, the scaling laws (39) for $G(t)$ imply corresponding results for $H$

$$
H(t)=c \underset{\sigma}{2} h_{ \pm}\left(t / t_{\sigma}\right) ; \quad \sigma \gtrless 0
$$

The $\sigma$-independent master functions $h_{ \pm}(\hat{t})$ are given by $\lambda, \xi, \zeta$. They can be evaluated from Eq. (46) with $G(t)$ replaced by $g_{ \pm}(\hat{t})$. Figure 14 exhibits the results for our model.

The properties of the functions $h_{ \pm}$can be understood by working out asymptotic expansions along the same lines as done before for $g_{ \pm}$[70]. The short-time expansion (41) yields a corresponding result for the new master functions

$$
h_{ \pm}(\hat{t})=\kappa(a) / \hat{t}^{2 a} \mp \widetilde{\kappa}(a)+O\left(\hat{t}^{2 a}\right)
$$

$$
\widetilde{\kappa}(x)=\frac{\kappa(x)\left[\Gamma(1+x) \Gamma(1-x)-\lambda^{2}\right] / \lambda-3 \xi+\zeta[2 \lambda+\Gamma(1+x) \Gamma(1-x)]}{2(1-\lambda)[\Gamma(1+x) \Gamma(1-x)-\lambda]} .
$$

For our model one finds $\widetilde{\kappa}(a)=2.48$. These results explain the deviations of $h_{ \pm}$from the critical law (47), which are shown in Fig. 14(a) for $\hat{t} \leqq 0.1$. For long times the glass correlator approaches a constant: $h_{+}(\hat{t} \rightarrow \infty)=(\xi-\lambda \zeta) /$ $(1-\lambda)^{2}$. Equation (42) leads to the long-time power-law divergence for the liquid correlator

$$
h_{-}(\hat{t})=\kappa(-b)\left(B \hat{t}^{b}\right)^{2}+\widetilde{\kappa}(-b)+O\left(\hat{t}^{-2 b}\right) .
$$

For our model one obtains $\widetilde{\kappa}(-b)=2.97$.

Substitution of the found scaling-law results into Eq. (36a) yields the results for the leading-plus-next-to-leading approximation of the correlator for $\sigma \gtrless 0$

$$
\begin{aligned}
\Phi_{q}(t)-f_{q}^{c}= & h_{q} c_{\sigma}\left\{g_{ \pm}\left(t / t_{\sigma}\right)+c_{\sigma}\left[h_{ \pm}\left(t / t_{\sigma}\right)\right.\right. \\
& \left.\left.+K_{q} g_{ \pm}\left(t / t_{\sigma}\right)^{2} \pm \overline{\bar{K}}_{q}\right]\right\} .
\end{aligned}
$$

For fixed rescaled times $\hat{t}=t / t_{\sigma}$, the corrections to the leading asymptotic laws vanish proportional to $\sqrt{|\epsilon|}$. The correction $c_{\sigma} h_{ \pm}(\hat{t})$ does not lead to a violation of the factorization theorem; it can be interpreted as a modification of the factor $G$ in Eq. (36a). The corrections to the factorization theorem are given by the terms $c_{\sigma} K_{q} g_{ \pm}^{2}$ and $c_{\sigma} \overline{\bar{K}}_{q}$. A corresponding result can be derived from Eq. (36b) for the dynamical susceptibility

$$
\chi_{q}(\omega)-\left(1-f_{q}^{c}\right)=h_{q} c_{\sigma}\left\{\left(z t_{\sigma}\right) g_{ \pm}\left(z t_{\sigma}\right)+c_{\sigma}\left[\left(z t_{\sigma}\right) h_{ \pm}\left(z t_{\sigma}\right)-K_{q}\left(z t_{\sigma}\right)^{2} g_{ \pm}\left(z t_{\sigma}\right)^{2} / \lambda+K_{q} / \lambda \mp \overline{\bar{K}}_{q}\right]\right\}
$$

The solid lines in Fig. 15(a) reproduce the decay curves from Fig. 4 for $n=9$, i.e., for reduced packing fractions $\epsilon= \pm 0.001$, and Fig. 15(b) shows the results for $n=6$, i.e., for $\epsilon= \pm 0.01$. Figure 16 exhibits the corresponding susceptibility spectra. The dashed lines in Figs. 15 and 16 show the scaling law approximation (37) and (39), while the dasheddotted lines exhibit the leading-plus-next-to-leadingapproximation results Eqs. (52).

The deviations of the asymptotic solutions from the correlator $\Phi_{q}(t)$ increase if the rescaled time $\hat{t}$ decreases to small values. But-according to Figs. 10(a) and 14(a)-for $\hat{t}<0.01$ all functions become very close to the results for the critical point. Therefore the results for these deviations can be inferred from the discussion for the $\epsilon=0$ case in Sec. VB. In Fig. 15-as opposed to in Fig. 8(a)—error symbols mark the absolute deviations of the analytic approximation from the full solutions by \pm 0.05 . The incorporation of the leading corrections extends the range of validity of the analytic formulas by about one order of magnitude. The figures confirm the conclusion which was drawn above from Fig. 8(a): Eq. (52a) describes the short-time dynamics except for that initial part, which is given by the free motion $\Phi_{q}^{(0)}(t)$. Similar results hold for the approximation of the high-frequency part of the susceptibility spectra. The analytical formulas work 


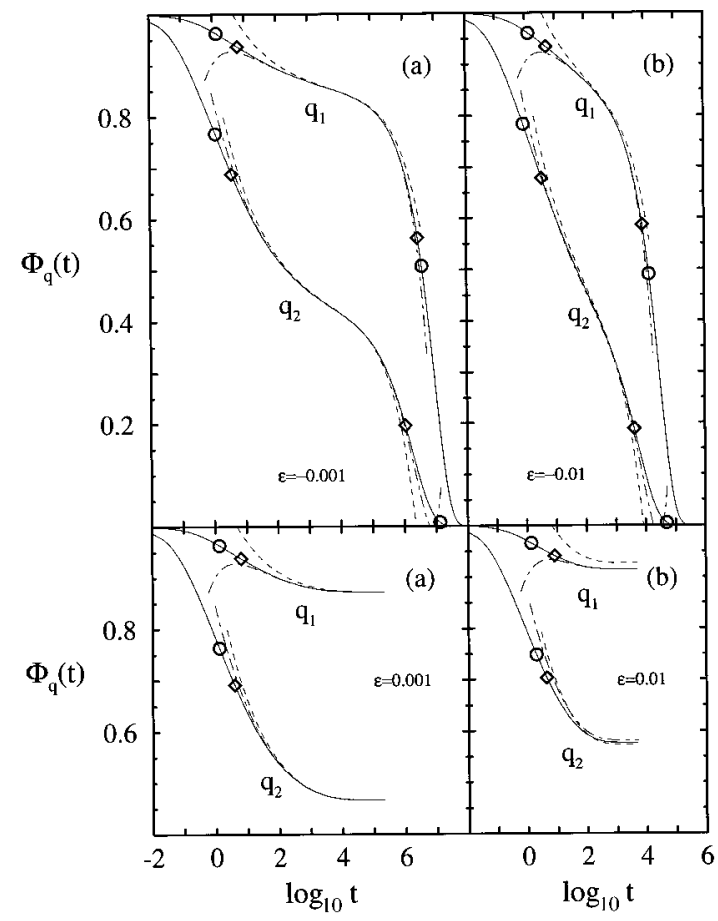

FIG. 15. The correlators from Fig. 4 for $\epsilon= \pm 10^{-3}[n=9$ in (a) $]$ and $\epsilon= \pm 10^{-2}[n=6$ in (b) $]$ are shown as solid lines. The dashed lines are the leading-order asymptotic $\beta$-relaxation results [Eqs. (37), (39), and (40)]; the diamonds mark the points where they deviate from the solution by \pm 0.05 . The dashed-dotted lines are the leading-plus-next-to-leading approximation Eq. (52a); the circles mark the points where they deviate from the solution by \pm 0.05 .

much better for the wave vector $q_{2}$ than for $q_{1}$. The reason was explained above in connection with Fig. 8. Notice in Fig. 16(a) that, for $q_{1}$ and $\epsilon=0.001$, the leading-order approximation deviates from the correct spectrum by more than $10 \%$ for all frequencies. A corresponding statement holds for $q_{1}$ and $q_{2}$, for the $\epsilon=0.01$ results in Fig. 16(b).

For the glass curves, the deviations of the approximations from the solutions decrease with increasing time. The corrections to the scaling-law asymptote of the long-time limit, Eq. (15), are much bigger for $q_{1}$ than for $q_{2}$. This is shown in Fig. 15 and was explained above in connection with Fig. 3. As before, one shows that the low-frequency spectra for the glass are regular. Therefore, one gets from Eq. (52a) the result: $\chi_{q}^{\prime \prime}(\omega)=h_{q} c_{\sigma}\left\{C_{0}+D_{q} c_{\sigma}+\mathcal{O}(\sigma)\right\}\left(\omega t_{\sigma}\right)+O\left(\omega^{3}\right)$ for $\epsilon>0$. The scaling-law coefficient $C_{0}$ is given by the regular low-frequency behavior of the master susceptibility $\hat{\chi}_{+}(\hat{\omega} \rightarrow 0) \sim C_{0} \hat{\omega}$. The leading corrections to the linear susceptibility spectrum are given by a coefficient, for which Eq. (52a) yields a $q$ dependence of the form: $D_{q}$ $=D+2 K_{q} C_{0} /(\lambda \sqrt{1-\lambda})$. For our model $D=-0.186$. The $q$ dependence of $K_{q}$, which was explained above in Sec. VC and which is exhibited in Fig. 2(c), is the reason, why the corrections are bigger for $q_{1}$ than for $q_{2}$ : $D_{1}=-1.94, D_{2}=-0.501$. These findings are demonstrated most clearly in Fig. 16(b).

The discussion of the liquid dynamics near the bifurcation singularity is more subtle than the one for the glass. This is due to the von Schweidler divergence for large $\hat{t}$, Eq. (42). In

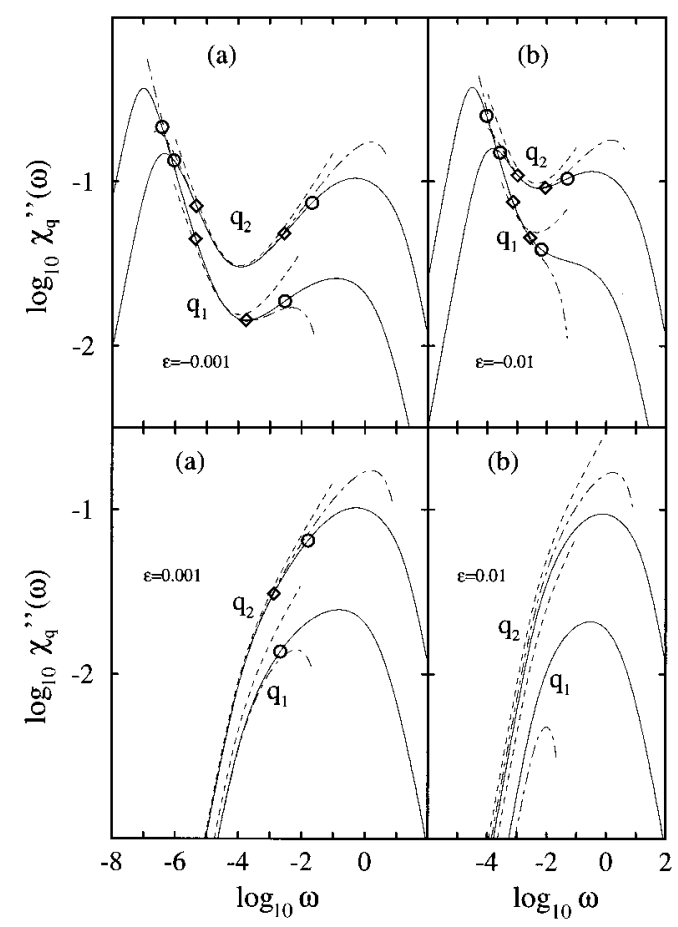

FIG. 16. The susceptibility spectra for the results in Fig. 15. Diamonds and circles mark the points of $10 \%$ deviations from the spectra for the leading and leading-plus-next-to-leading asymptotic formulas, respectively. Notice that for $\epsilon=0.01$ the approximations deviate for all frequencies by more than $10 \%$ from the solutions.

the expression $c_{\sigma} B\left(t / t_{\sigma}\right)^{b}$, one can combine the power laws for $c_{\sigma}$ and $\left(1 / t_{\sigma}\right)$ Eq. (40), to a new power-law time scale

$$
t_{\sigma}^{\prime}=t_{0}^{\prime} /|\sigma|^{\gamma}, \quad t_{0}^{\prime}=t_{0} / B^{1 / b}, \quad \gamma=\frac{1}{2 a}+\frac{1}{2 b} .
$$

For our model one finds: $t_{0}^{\prime}=0.578, \gamma=2.46$. Remembering the von Schweidler law $g_{-} \sim-B \hat{t}^{b}$ and the corresponding result $h_{-} \sim \kappa(-b)\left(B \hat{t}^{b}\right)^{2}$ from Eq. (51), one obtains from Eq. (52a) for the asymptote of the $\beta$-relaxation liquid correlator for large $\hat{t}=t / t_{\sigma}$

$$
\begin{gathered}
\Phi_{q}(t)-f_{q}^{c}=-h_{q}\left(t / t_{\sigma}^{\prime}\right)^{b}\left\{1-\left[K_{q}+\kappa(-b)\right]\left(t / t_{\sigma}^{\prime}\right)^{b}\right\}, \\
\epsilon<0 .
\end{gathered}
$$

On the other hand, in Sec. VC, the solutions have been considered for $\epsilon \rightarrow 0-$ for fixed $\widetilde{t}=t / t_{\sigma}^{\prime}$. If one compares the result (30a) with Eq. (54), one identifies the previously unspecified time scale $t_{\sigma}^{\prime}$ for the $\alpha$ process with the power-law scale from Eq. (53).

According to the preceding paragraph, the relaxation of the liquid for times long on scale $t_{\sigma}, \hat{t}=t / t_{\sigma} \gg 1$, is identical with the relaxation below the plateau $f_{q}^{c}$ for times short on scale $t_{\sigma}^{\prime}, \tilde{t}=t / t_{\sigma}^{\prime} \ll 1$. The relation of the analytic $\beta$-relaxation results to the solutions for large $\hat{t}$ and small $\hat{\omega}$ can therefore be inferred from the discussion of Fig. 9 in Sec. VC.

The scaling-law result describes the decay of $\Phi_{2}(t)$ near the plateau for $\epsilon=-0.001$ well for a time window of about 
five and a half decades, as follows from Fig. 15(a). The approximation of the minimum of $\chi_{2}^{\prime \prime}(\omega)$ by the master spectrum only works for a three decade frequency window, as shown in Fig. 16(a). A similar observation holds for the leading-plus-next-to-leading asymptotic results: For $\epsilon=-0.01$, the decay of $\Phi_{1}(t)$ is described for a time window of about four decades, as shown in Fig. 15(b). Figure 16(b) demonstrates that the corresponding approximation for the susceptibility spectrum works for a frequency window of two decades only. The window for the applicability of the asymptotic laws is larger in the time domain than in the frequency domain. This implies that it is easier to identify the scaling laws for $\Phi_{q}(t)$ versus $\log _{10} t$ diagrams than for $\log _{10} \chi_{q}^{\prime \prime}(\omega)$ versus $\log _{10} \omega$ graphs. The reason is the appearance of the factors $k_{a}$ and $k_{b}$ for the correction terms in Eqs. (27b) and (30b) as was explained in Secs. VB and VC, respectively.

\section{THE SECOND SCALING-LAW REGIME}

The first scaling law and its leading corrections deal with the dynamics on scale $t_{\sigma}$, Eq. (52a). For $\varphi>\varphi_{c}$, the correlators arrest for $t / t_{\sigma} \gg 1$ and therefore the results of Secs. III and VI provide a complete description of the MCT bifurcation dynamics of the glass state for small $\varphi-\varphi_{c}$. However, for $\varphi<\varphi_{c}$, there is the second relaxation step towards equilibrium, which deals with the dynamics on scale $t_{\sigma}^{\prime}$. After yet another reformulation of the equations of motion (Sec. VII A), a scaling law for the $\alpha$ process-the superposition principle-will be derived as leading-order asymptotic result (Sec. VII B). Then leading corrections to this law will be discussed in Sec. VII C.

\section{A. The equations of motion for the $\alpha$ process near the critical point}

Let us introduce rescaled times $\tilde{t}$ and frequencies $\widetilde{z}, \widetilde{\omega}$ in the scale invariant equation (19) as done before $\widetilde{t}=t / t_{\sigma}^{\prime}, \widetilde{z}=z t_{\sigma}^{\prime}, \widetilde{\omega}=\omega t_{\sigma}^{\prime}$. To study the dynamics on scale $t_{\sigma}^{\prime}$, we carry out an asymptotic expansion for small negative reduced packing fractions $\epsilon$

$$
\Phi_{q}(t)=\widetilde{\Phi}_{q}(\widetilde{t})+\epsilon\left(1-f_{q}^{c}\right)^{2} \widetilde{\Psi}_{q}(\widetilde{t})+O\left(\epsilon^{2}\right)
$$

The expansion of the mode-coupling functional, Eq. (2), is given by the following coefficients:

$$
\begin{gathered}
C_{q}(\widetilde{t})=\mathcal{F}_{q}^{c}(\widetilde{\Phi}(\widetilde{t})), \quad C_{q}^{\prime}(\widetilde{t})=\partial \mathcal{F}_{q}^{c}(\widetilde{\Phi}(\widetilde{t})) / \partial \epsilon \\
C_{q k}(\widetilde{t})=\left[\partial \mathcal{F}_{q}^{c}(\widetilde{\Phi}(\widetilde{t})) / \partial f_{k}\right]\left(1-f_{k}^{c}\right)^{2} .
\end{gathered}
$$

Because of $\widetilde{\Phi}_{q}(\widetilde{t \rightarrow 0})=f_{q}^{c}$, these functions have as initial values the numbers $C_{q}^{c}, C_{q}^{\prime c}$ and $C_{q k}^{c}$, respectively, which have been introduced in Eqs. (9). Specializing Eq. (19) to $\epsilon=0$, one finds the nonlinear equation of motion for the leading-order contribution $\widetilde{\Phi}_{q}[68]$

$$
\widetilde{\Phi}_{q}(\widetilde{z}) /\left[1+\widetilde{z} \widetilde{\Phi}_{q}(\widetilde{z})\right]=C_{q}(\widetilde{z})
$$

For the leading correction $\widetilde{\Psi}_{q}$ the linear equation of motion is obtained

$$
\begin{aligned}
& \left(1-f_{q}^{c}\right)^{2} \widetilde{\Psi}_{q}(\widetilde{z}) /\left[1+\widetilde{z} \widetilde{\Phi}_{q}(\widetilde{z})\right]^{2} \\
& \quad=C_{q}^{\prime}(\widetilde{z})+\sum_{k} \mathcal{L}\left[C_{q k}(\widetilde{t}) \widetilde{\Psi}_{k}(\widetilde{t})\right](\widetilde{z}) .
\end{aligned}
$$

\section{B. The leading-order results}

If one ignores terms of order $\epsilon$, one gets from Eq. (55) scaling laws for the correlators and susceptibilities

$$
\Phi_{q}(t)=\widetilde{\Phi}_{q}\left(t / t_{\sigma}^{\prime}\right) ; \quad \chi_{q}(\omega)=\widetilde{\chi}_{q}\left(\omega t_{\sigma}^{\prime}\right)
$$

The control-parameter-independent master functions $\widetilde{\Phi}_{q}(\widetilde{t})$ and $\widetilde{\Phi}_{q}(\widetilde{z})$ are to be determined from Eq. (57), which is the Laplace transform of Eq. (29) from Sec. V C. The susceptibility master function is related to the Laplace transform of the correlator by: $\widetilde{\chi}_{q}(\widetilde{\omega})=\widetilde{z} \widetilde{\Phi}_{q}(\widetilde{z})+f_{q}^{c}, \widetilde{z}=\widetilde{\omega}+\mathrm{i} 0$. Within the leading-order description, the sensitive dependence of the long-time or low-frequency liquid dynamics is thus caused entirely by the singularly varying time scale $t_{\sigma}^{\prime}$ from Eq. (53).

If the correlators $\Phi_{q}$ for the $\alpha$ process are considered as functions of the rescaled times $\widetilde{t}$, they coincide for all $\epsilon$ with the common master curve $\widetilde{\Phi}_{q}$. Equivalently, if the susceptibilities $\chi_{q}$ are considered as functions of the rescaled frequencies $\widetilde{\omega}$, they are superimposed for all control parameters $\varphi$ onto the common master susceptibility $\widetilde{\chi}_{q}$. Therefore, the scaling laws (59) are referred to as the superposition principle, which is a concept from the glass literature [1]. This principle is obtained as leading asymptotic result for the MCT $\alpha$ process in the limit $\varphi \rightarrow \varphi_{c}-$. The precise formulation is the interval of rescaled times $\widetilde{t}$, where the $\Phi_{q}(\widetilde{t})$ can be superimposed on $\widetilde{\Phi}_{q}(\widetilde{t})$, expands to arbitrary sizes for $\epsilon \rightarrow 0-$. This is demonstrated in Fig. 17 for our model. Similarly, the interval of rescaled frequencies $\widetilde{\omega}$, where $\chi_{q}(\widetilde{\omega})$ coincides with the master susceptibilities $\widetilde{\chi}_{q}(\widetilde{\omega})$, expands for decreasing $-\epsilon$, as is shown in Fig. 18. The rescaling, i.e., the replot of the results as functions of $\log _{10} \widetilde{t}$ or $\log _{10} \widetilde{\omega}$ instead of as functions of $\log _{10} t$ or $\log _{10} \omega$, respectively, is done by a shift of the curves parallel to the abscissa by $\pm \log _{10} t_{\sigma}^{\prime}$.

In Sec. IV, $\alpha$-relaxation scales $\tau_{q}^{\prime}=1 / \omega_{\max }(q)$ and $\bar{\tau}_{q}^{\prime}$ have been introduced. Let us define in a similar manner $\widetilde{\omega}_{\text {max }}(q)$ as the peak position of the $\widetilde{\chi}_{q}^{\prime \prime}$ versus $\widetilde{\omega}$ graph and $\widetilde{\tau}_{q}^{\prime}$ by $\widetilde{\Phi}_{q}\left(\widetilde{\tau}_{q}^{\prime}\right)=f_{q}^{c} / 2$. For our model, one finds for $q_{1}$ : $\widetilde{\omega}_{\max 1}=0.474, \widetilde{\tau}_{1}^{\prime}=1.17$ and for $q_{2}: \quad \widetilde{\omega}_{\max 2}=2.16, \widetilde{\tau}_{2}^{\prime}$ $=0.205$. Within the range of validity of the leading-order result (59), one gets

$$
\tau_{q}^{\prime}=t_{\sigma}^{\prime} / \widetilde{\omega}_{\max }(q) ; \quad \bar{\tau}_{q}^{\prime}=t_{\sigma}^{\prime} \widetilde{\tau}_{q}^{\prime}
$$

The superposition principle explains that there is only one $\alpha$ scale $t_{\sigma}^{\prime}$. The scales $\tau_{q}^{\prime}$ or $\bar{\tau}_{q}^{\prime}$ quantify the slowing down of the dynamics upon freezing. These scales depend on the correlator under discussion. But different scales differ only by $\epsilon$-independent prefactors $1 / \widetilde{\omega}_{\max }(q)$ and $\widetilde{\tau}_{q}$, respectively. In Fig. $7(\mathrm{a})$, the asymptotic power law for $\tau_{2}^{\prime}$ is shown to describe the solution well for $n \geqslant 5$. The corresponding statement is demonstrated in Figs. 13(a) and 13(b) for $\omega_{\max 2}$. 




FIG. 17. Correlators $\Phi_{q}$ for the reduced packing fractions $\epsilon=-10^{-n / 3}, n=1,3,5,7,9$ as function of the rescaled time $\widetilde{t}=t / t_{\sigma}^{\prime}$ with $t_{\sigma}^{\prime}$ given by Eq. (53) (solid lines). The thick solid lines are the $\alpha$-relaxation master functions $\widetilde{\Phi}$ obtained from Eq. (29). The dashed lines are the short-time parts of the leading-plus-nextto-leading approximation for the $\alpha$ correlators $\widetilde{\Phi}_{q}(\widetilde{t})+h_{q} B_{1} \sigma \widetilde{t}^{-b}$.

It was discussed in Sec. V C that the $\alpha$ process deals with a crossover from the von Schweidler short $\tilde{t}$ expansion to exponential decay at long times. As a result the stretched $\alpha$ peak of the susceptibility spectrum comes about due to the crossover from a regular low $\widetilde{\omega}$ spectrum to a von Schweidler high $\widetilde{\omega}$ decay. The $\alpha$-relaxation times $\tau_{q}$ or $\bar{\tau}_{q}$ exhibit characteristic variations as a function of the wave vector $q$ and so do the stretching properties, as has been discussed comprehensively for the hard-sphere system in Ref. [59].

\section{The leading correction to the second scaling law}

It was shown in Sec. VIC that the leading corrections to the first scaling-law results are of relative order $|\epsilon|^{1 / 2}$. If we stick to the same degree of accuracy for the description of the $\alpha$ process we can use Eq. (59). There are no corrections to the second scaling-law results of order $|\epsilon|^{1 / 2}$. For fixed $\widetilde{t}$, the corrections to the second scaling law are only of order $|\epsilon|$. This observation explains, why the implications of Eq. (59) still hold for such large $\epsilon$, where the first scaling-law results are no longer applicable. For example, one infers from Fig. 18 that the second scaling-law results describe a major part of the $\alpha$ process for $\epsilon=-10^{-5 / 3}$ and $q=q_{1}$. On the other hand, it follows from Fig. 16(b) that the first scaling law is only of limited use, if at all, to describe the spectra of the $\beta$ process for $\epsilon=-10^{-6 / 3}$.

With decreasing $\widetilde{t}$, the functions $\Phi_{q}(\widetilde{t})$ increase above the maximum $f_{q}^{c}$ of $\widetilde{\Phi}_{q}(\widetilde{t})$ as is shown in Fig. 17. Similarly, with

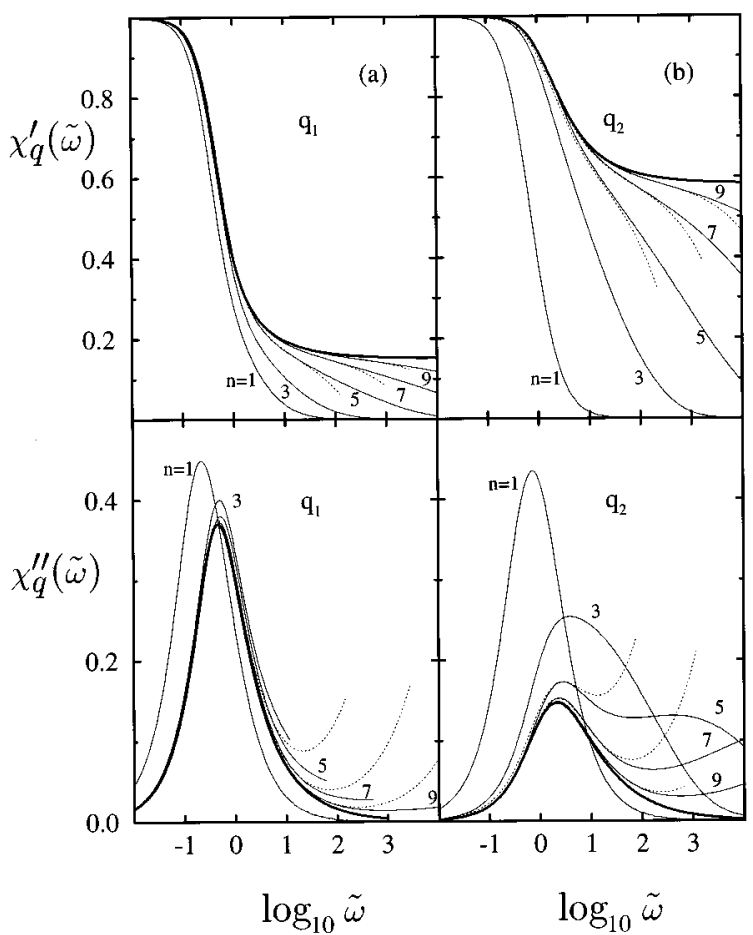

FIG. 18. Susceptibilities for the results from Fig. 17 as functions of the rescaled frequency $\widetilde{\omega}=\omega t_{\sigma}^{\prime}$.

increasing $\widetilde{\omega}$ the rescaled susceptibility functions $\chi_{q}^{\prime \prime}(\widetilde{\omega})$ or $\chi_{q}^{\prime}(\widetilde{\omega})$ increase above the master spectra or decrease below the plateau $1-f_{q}^{c}$, respectively, as is demonstrated in Fig. 18. These deviations from the second scaling laws for times short on scales $t_{\sigma}^{\prime}$ or frequencies large on scale $1 / t_{\sigma}^{\prime}$, respectively, are caused by the correction term $\epsilon\left(1-f_{q}^{c}\right)^{2} \widetilde{\Psi}_{q}$ in Eq. (55). The equation of motion (58) can be solved for small $\widetilde{t}$ by expansions in powers of $\vec{t}^{b}$, following the same procedure as explained in the preceding sections. One gets

$$
\Phi_{q}(t)=\widetilde{\Phi}_{q}(\widetilde{t})+h_{q} B_{1} \sigma \widetilde{t}^{-b}+\sigma O\left(\widetilde{t}^{0}, \sigma\right)
$$

The critical amplitude $h_{q}$ is the one from the factorization theorem (37) and coefficient $B_{1}$ occured in Eq. (42) during the discussion of the first scaling-law results. The dashed lines for short $\tilde{t}$ in Fig. 17 exhibit the sum of the leading contribution $\widetilde{\Phi}_{q}(\widetilde{t})$ and the second term of Eq. (61) as dashed lines for short $\widetilde{t}$; Fig. 18 shows the corresponding results for the susceptibilities. It has been explained in Sec. III that the ratio $h_{q} / f_{q}^{c}$ is smaller for wave vectors near the structure-factor-peak position than for $q$ off this value [compare Fig. 2(b)]. This observation explains why the second scaling law works better for $q_{1}$ than for $q_{2}$.

Let us consider the result $(61)$ for small $\widetilde{t}$. Then, the $\sigma O\left(\widetilde{t}^{0}\right)$ term can be neglected and the scaling function $\widetilde{\Phi}_{q}(\widetilde{t})$ can be replaced by the von Schweidler law, i.e., by the leading terms in Eq. (30a)

$$
\Phi_{q}(t)=f_{q}^{c}-h_{q}\left\{\widetilde{t}^{b}-B_{1} \sigma \widetilde{t}^{-b}\right\}
$$


If one remembers the definitions of $t_{\sigma}^{\prime}$ in Eq. (53) and of $c_{\sigma}, t_{\sigma}$ in $\mathrm{Eq}(40)$, one can rewrite the result as

$$
\Phi_{q}(t)=f_{q}-h_{q} c_{\sigma}\left\{B \hat{t}^{b}-B_{1} /\left(\hat{t}^{b} B\right)\right\},
$$

where $\hat{t}=t / t_{\sigma}$. It was known from a previous work [68] that the leading expansion of the $\alpha$ correlator for $\widetilde{t} \ll 1, \Phi_{q}(t) \sim f_{q}^{c}-h_{q} \widetilde{t}^{b}$, agrees with the leading expansion of the first scaling law for $\hat{t} \gg 1, \Phi_{q}(t) \sim f_{q}^{c}-h_{q} c_{\sigma} B \hat{t}^{b}$. The formulas (62) extend these findings so that the next-to-leading terms are included. The correction of order $\sigma$ to the second scaling law for times short on scale $t_{\sigma}^{\prime}$ is given by the last term in Eq. (62a). And this agrees with the next-to-leading term for times long on scale $t_{\sigma}$, as given by the expansion of the first scaling law in Eq. (42).

\section{SUMMARY AND CONCLUSIONS}

In this paper we presented a comprehensive discussion of the $\alpha$ - and $\beta$-scaling laws, which are two of the central predictions of the MCT. As a result of a bifurcation analysis the factorization property can be derived: In the $\beta$-relaxation region the time dependence of the correlation functions is given by a single function $G$ which obeys a one-parameter scaling law, Eqs. (39) and (40). The wave-vector dependence enters only via the critical form factor $f_{q}^{c}$ and the critical amplitude $h_{q}$. In Figs. 11 and 12 the rescaled correlators and susceptibility spectra of the hard-sphere model are compared with the master function. The diamonds mark the time and frequency windows were the scaled curves are well described by the asymptotic formulas. Let us emphasize that Figs. 11 and 12 present a hard test of the asymptotic laws, because all the parameters that enter Eqs. (37)-(40), except a single time scale $t_{0}$, have been calculated not fitted. These figures can be easily interpreted if one considers that the $\beta$-scaling law is the leading order of an asymptotic expansion about the critical plateau $f_{q}^{c}$ in the vicinity of a glass transition. This implies that for a system near a glass transition singularity the scaled curves should collapse onto the master function as soon as they come close to $f_{q}^{c}$. If the system is moved towards the glass transition the window where the correlators remain near the plateau expands-and so does the range of validity of the factorization theorem and of the scaling laws. The verification of this behavior is an essential part of a proper MCT analysis of experimental data (see, e.g., Refs. $[19,20]$ ).

The factorization theorem does not hold uniformly with respect to wave-vector variations. For a given wave-vector modulus $q$ and a given number of time decades, there is a positive $\epsilon_{0}$, so that Eqs. (37)-(40) hold for all reduced packing fractions $\epsilon=\left(\varphi-\varphi_{c}\right) / \varphi_{c}$, with $|\epsilon|<\epsilon_{0}$. However, for $\epsilon$ $\neq 0$ no matter how small $|\epsilon|$ may be, one can find a $q$ so small, that the $\beta$-scaling law becomes invalid, because it is tarnished by the hydrodynamic singularities. For our hardsphere colloid model we found that the $\beta$-relaxation scaling laws describe the dynamics for a wave-vector band $q_{0} \leqslant q \leqslant q_{3}$ and $|\epsilon| \leqslant 10^{-4}$ in a time window of more than five orders of magnitude.

The arguments given above for the correlators can be applied to the spectra in Fig. 12, if one notices that the region close to the plateau corresponds to the region in the vicinity of the minimum or the knee for liquid or glass curves, respectively. Again, it is important for an experimental test of the theory to demonstrate that the range of validity shrinks with increasing distance from the glass transition (see, e.g., Refs. $[11,31,32])$. For the wave-vector band and the packing fractions specified above, $\beta$ scaling holds for more than three decades in the frequency domain. The factorization of the spectra implies, for example, that the position of the minima of various susceptibility spectra should coincide. This property was verified for a set of four representative wave vectors by neutron-scattering studies for the mixed salt CKN [71]. Within MCT, one can show that the susceptibilities $\chi_{A}(z)$ for all variables $A$ which couple to density fluctuations obey the factorization theorem as asymptotic law [37]. Indeed it was shown [39] that the susceptibility spectra $\chi_{A}^{\prime}(\omega)$ obtained by depolarized light scattering for $\mathrm{CKN}$, exhibit minima in agreement with the ones reported for neutron scattering. Apparently for the cited system and the mentioned probing variables, the preasymptotic corrections are rather small. Minima positions for Salol as obtained by light scattering have been compared with those obtained by neutron scattering in Ref. [33]. In that case, the results were similar to what was discussed for Fig. 13(a): for large temperature $T$ the minimum positions differed but they converged to the same frequencies upon lowering $T$ towards the critical value $T_{c}$.

Figures 11-13 demonstrate that the correlators deviate differently from the master curves, depending on the wave vector, and therefore the window of validity of the $\beta$-scaling law is $q$ dependent. This effect can be seen even more dramatically for the susceptibilities shown in Fig. 16(a) for $\epsilon=-0.001$ : The minimum positions and the slopes agree reasonably well for $\omega \leqslant \omega_{\min }$. For $\omega>\omega_{\min }$, the spectrum for $q_{2}$ follows the master spectrum for more than a decade frequency increase. However, the spectrum for $q_{1}$ differs strongly from the leading result for $\omega>\omega_{\min }$; it looks as if there was a critical spectrum specified by an effective exponent $a_{e f f}$, which is considerably smaller than the value $a$. For $\epsilon=-0.01$, the susceptibility $\chi_{1}^{\prime \prime}(\omega)$ in Fig. 16(b) does not exhibit a minimum at all, even though there is a well developed $\alpha$ peak. The anomaly noticed for $\epsilon=-0.001$ can thus be understood as precursor for the disappearance of the minimum for large separations from the critical point. Notice that the minimum shape for the susceptibility $\chi_{2}^{\prime \prime}(\omega)$ can still be described well by the scaling-law master spectrum for $\epsilon=-0.01$.

The need to broaden our understanding of the deviations from the $\beta$-scaling law made it appear worthwhile to push the asymptotic expansion to the next-to-leading order. We could demonstrate that the leading corrections extend the range of validity of the first-order approximation at and off the critical point substantially and can therefore serve as an explanation for the deviations mentioned above (see Figs. 3, $8,9,15$, and 16). They can explain, in particular, the shift [Fig. 16(a)] and the disappearance [Fig. 16(b)] of the susceptibility minimum.

The appearance of wave-vector dependent amplitudes $K_{q}$ and $\bar{K}_{q}$ in the next-to-leading-order terms, Eqs. (52), implies that the corrections spoil the factorization property, i.e., 
the deviations from the scaling law results are $q$ dependent. Close to the glass transition singularity the dominant effects are the short-time corrections to the critical law and the longtime corrections to the von Schweidler law, which were discussed in Secs. V A, V B. This knowledge has also been used to explain why the window of validity of the $\beta$-scaling law is much smaller in the frequency domain than in the time domain. This effect, noticed previously by Kob and Andersen in connection with a MCT analysis of computer simulation data (see Fig. 14 in Ref. [72]), depends only on the value of the exponent parameter $\lambda$. For our model the windows differ by nearly two orders of magnitude.

Further, we could prove that the long-time corrections are connected with the short-time corrections [Eqs. (27) and (30)]. As a consequence, the numbers labeling the scaled correlators in Fig. 11 are in the same order from top to bottom on the short-time side as on the long-time side. Experiments on hard-sphere colloids are consistent with this conclusion (see Fig. 14 in Ref. [20]). Suppose corrections to the scaling law have been measured for some correlator for short and long times. With Eqs. (27) and (30) one can then determine the number $\kappa(a)-\kappa(-b)$. If one now measures the short-time corrections for some other correlator, say $\Phi_{q}$, one can determine $K_{q}+\kappa(a)$. Then one knows $K_{q}+\kappa(-b)$ and thereby the long-time corrections. Consequently, our results provide a quantitative prediction which holds for every MCT model. Analogous results hold for the susceptibility spectra in Fig. 12.

The corrections depend on the details of the modecoupling functional of the investigated system via the parameters $K_{q}, \bar{K}_{q}, \zeta, \xi$, and $\kappa$ just as the leading-order results depend on the particular model via $f_{q}^{c}, h_{q}$, and $\lambda$. This is since the cage effect, which is the common origin for the bifurcation scenario predicted by the MCT, depends on the short-distance interactions between the atoms or molecules. Nevertheless, we were able to explain the general tendencies for variations with $q$ displayed by $f_{q}^{c}, h_{q}$, and $K_{q}$, and thereby reach a qualitative understanding of the results for the hard-sphere model. The theory can be trivially extended to one dealing with mixtures [68], and one can obviously generalize the formulas for the leading corrections in the same way as it has been done in the case of the leading-order results [73]. It would be desirable to carry out an explicit discussion of the formulas for representative mixtures in order to see whether a qualitative understanding of mixture results can be achieved as well.

The corrections to the $\alpha$-scaling law cannot be calculated in the same manner as the corrections to the $\beta$-scaling law. We could only derive analytic formulas for the short-time corrections, which are in fact identical to the short-time corrections to the von Schweidler law of the $\beta$-scaling theory. Despite these difficulties a general result can be found: The leading-order corrections to $\alpha$ scaling are of higher order in the separation parameter than the leading corrections to $\beta$ scaling. That is why the superposition principle still works quite well for separations where the factorization property does not hold any more (compare the $n=7$ and $n=9$ results in Fig. 18 with the $n=8$ ones in Fig. 12).

All results in this paper have been obtained from Eq. (19), which deals with a dynamical regime where transient effects have died out. The derived analytical formulas for the solution of the MCT equations of motion deal with structural relaxation only, which, except for the time scale $t_{0}$, is entirely determined by the mode-coupling functional, Eq. (2). However, there may be interesting slow dynamical processes due to the transient, which can interfere with the structural relaxation. Thereby, they can also cause preasymptotic corrections to the bifurcation dynamics. But these corrections are not considered in our paper. Studying the crossover from slow transient dynamics to the structural relaxation may be important for an interpretation of data or of MCT solutions. The hydrodynamic singularities which were mentioned above, are obvious examples for such preasymptotic corrections. Let us also mention two less obvious ones. First, an additional time fractal, expected for hard-sphere systems, had to be incorporated to interpret within the MCT formalism the shear dynamics of a colloidal suspension [21]. Second, conventional liquids exhibit phononlike excitations $[51,52]$, whose bare dispersion law enters the MCT equation of motion, Eq. (1), as $\Omega_{q}$. MCT predicts that such excitations are also present in the glass $[36,68]$. These oscillations may mask, for example, the critical decay, as was discussed in Ref. [14].

The concept of spontaneous arrest of fluctuations in a disordered system is an idealization. The singular transition to an ideal glass state, as obtained within the idealized MCT, results from the assumption, that fluctuating forces couple to density-fluctuation pairs whose motion can be treated by a factorization ansatz. If one takes into account also coupling to currents, one obtains an extended MCT, where ergodicity is restored for all $T$ and $n$ so that correlation functions always relax to zero $[74,75]$. The current modes play the role of phonons and the indicated relaxation mechanism approximately describes transport via phonon-assisted hopping. As a result, the bifurcation singularity is bypassed and the singular transition is replaced by a continuous crossover. There still is a $\beta$ regime, where the factorization theorem (37) describes the dynamics in leading order. But the function $G(t)$ now is to be calculated from a two-parameter scaling law $[75,76]$. This extended $\beta$-relaxation theory has been used $[32,77,78]$ for data interpretation with the intention to explain deviations from the scaling laws of the idealized MCT. The avoided singularity of the extended MCT does not anymore separate liquid states from ideal glass states, but still is the origin of the stretched dynamics and the strong density or temperature dependence of the time scales. The extended $\beta$-scaling law explains the crossover from the dynamics typical for disordered solid, i.e., thermally activated processes, to dynamics characteristic for a liquid, i.e., cage and backflow effects. At present, it is unclear whether our methods can also be applied to derive, within the extended MCT, preasymptotic correction formulas for the mentioned twoparameter scaling law.

\section{ACKNOWLEDGMENTS}

We cordially thank Herman Cummins and Walter Kob for many stimulating discussions and for helpful comments on our manuscript. Our work was supported by Verbundprojekt BMBF 03-G04TUM. M.F. acknowledges financial support by the Deutsche Forschungsgemeinschaft under Grant No. Fu 309/1-1. 
[1] J. Wong and C. A. Angell, Glass: Structure by Spectroscopy (Dekker, New York, 1976).

[2] R. Kohlrausch, Pogg. Ann. Phys. Chem. (Leipzig) 91, 56 (1854).

[3] E. Ritter von Schweidler, Ann. Phys. (Leipzig) 24, 711 (1907).

[4] K. S. Cole and R. H. Cole, J. Chem. Phys. 9, 341 (1941).

[5] M. Fuchs, J. Non-Cryst. Solids 172-174, 241 (1994).

[6] W. Götze, Z. Phys. B 56, 139 (1984).

[7] W. Götze, Z. Phys. B 60, 195 (1985).

[8] W. Götze and L. Sjögren, J. Phys. Condens. Matter 1, 4183 (1989).

[9] W. Knaak, F. Mezei, and B. Farago, Europhys. Lett. 7, 529 (1988).

[10] N. J. Tao, G. Li, and H. Z. Cummins, Phys. Rev. Lett. 66, 1334 (1991).

[11] G. Li, W. M. Du, X. K. Chen, H. Z. Cummins, and N. J. Tao, Phys. Rev. A 45, 3867 (1992).

[12] J. Wuttke, J. Hernandez, G. Li, G. Coddens, H. Z. Cummins, F. Fujara, W. Petry, and H. Sillescu, Phys. Rev. Lett. 72, 3052 (1994).

[13] J. Wuttke, W. Petry, G. Coddens, and F. Fujara, Phys. Rev. E 52, 4026 (1995).

[14] T. Franosch, W. Götze, M. R. Mayr, and A. P. Singh, Phys. Rev. E 55, 3183 (1997).

[15] D. Sidebottom, R. Bergman, L. Börjesson, and L. M. Torrell, Phys. Rev. Lett. 71, 2260 (1993).

[16] W. van Megen and P. N. Pusey, Phys. Rev. A 43, 5429 (1991).

[17] W. Götze and L. Sjögren, Phys. Rev. A 43, 5442 (1991).

[18] E. Bartsch, M. Antonietti, W. Schupp, and H. Sillescu, J. Chem. Phys. 97, 3950 (1992).

[19] W. van Megen and S. M. Underwood, Phys. Rev. Lett. 70, 2766 (1993).

[20] W. van Megen and S. M. Underwood, Phys. Rev. E 49, 4206 (1994).

[21] T. G. Mason and D. A. Weitz, Phys. Rev. Lett. 75, 2770 (1995).

[22] E. Leutheusser, Phys. Rev. A 29, 2765 (1984).

[23] U. Bengtzelius, W. Götze, and A. Sjölander, J. Phys. C 17, 5915 (1984).

[24] S. P. Das, G. F. Mazenko, S. Ramaswamy, and J. J. Toner, Phys. Rev. Lett. 54, 118 (1985).

[25] F. Mezei, Ber. Bunsenges. Phys. Chem. 95, 1118 (1991).

[26] I. C. Halalay, Y. Yang, and K. A. Nelson, J. Non-Cryst. Solids 172-174, 175 (1994).

[27] J. Ma, D. V. Bout, and M. Berg, Phys. Rev. E 54, 2786 (1996).

[28] J. Baschnagel, Phys. Rev. B 49, 135 (1994).

[29] W. Kob and H. C. Andersen, Phys. Rev. E 51, 4626 (1995).

[30] P. Gallo, F. Sciortino, P. Tartaglia, and S.-H. Chen, Phys. Rev. Lett. 76, 2730 (1996).

[31] G. Li, W. M. Du, A. Sakai, and H. Z. Cummins, Phys. Rev. A 46, 3343 (1992).

[32] W. M. Du, G. Li, H. Z. Cummins, M. Fuchs, J. Toulouse, and L. A. Knauss, Phys. Rev. E 49, 2192 (1994).

[33] J. Toulouse, R. Pick, and C. Dreyfus, Mater. Res. Soc. Symp. Proc. 407, 161 (1996).

[34] N. Menon, K. P. O'Brien, P. Dixon, L. Wu, S. R. Nagel, B. D. Williams, and J. Carini, J. Non-Cryst. Solids 141, 61 (1992).

[35] P. Lunkenheimer, A. Pimenov, M. Dressel, Y. G. Goncharov, R. Böhmer, and A. Loidl, Phys. Rev. Lett. 77, 318 (1996).

[36] W. Götze, in Liquids, Freezing and Glass Transition, edited by
J. P. Hansen, D. Levesque, and J. Zinn-Justin (North-Holland, Amsterdam, 1991), p. 287.

[37] W. Götze and L. Sjögren, Rep. Prog. Phys. 55, 241 (1992).

[38] R. Schilling, in Disorder Effects on Relaxational Processes, edited by R. Richert and A. Blumen (Springer-Verlag, Berlin, 1994), p. 193.

[39] H. Z. Cummins, G. Li, W. M. Du, and J. Hernandez, Physica A 204, 169 (1994).

[40] H. Z. Cummins, G. Li, W. M. Du, J. Hernandez, and N. J. Tao, Transp. Theory Stat. Phys. 24, 981 (1995).

[41] I. C. Halalay, Y. Yang, and K. A. Nelson, Transp. Theory Stat. Phys. 24, 1053 (1995).

[42] L. M. Torell, L. Börjesson, and A. P. Sokolov, Transp. Theory Stat. Phys. 24, 1097 (1995).

[43] W. van Megen, Transp. Theory Stat. Phys. 24, 1017 (1995).

[44] E. Bartsch, Transp. Theory Stat. Phys. 24, 1125 (1995).

[45] W. Petry and J. Wuttke, Transp. Theory Stat. Phys. 24, 1075 (1995).

[46] J.-P. Hansen and S. Yip, Transp. Theory Stat. Phys. 24, 1149 (1995).

[47] W. Kob and H. Andersen, Transp. Theory Stat. Phys. 24, 1179 (1995).

[48] J. Baschnagel, Transp. Theory Stat. Phys. 24, 1249 (1995).

[49] W. Götze and L. Sjögren, J. Math. Analysis Appl. 195, 230 (1995).

[50] D. Forster, Hydrodynamic Fluctuations, Broken Symmetry, and Correlation Functions (Benjamin, Reading, MA, 1975).

[51] J.-P. Boon and S. Yip, Molecular Hydrodynamics (McGrawHill, New York, 1980).

[52] J.-P. Hansen and I. R. McDonald, Theory of Simple Liquids, 2nd ed. (Academic, London, 1986).

[53] K. Kawasaki, Phys. Rev. 150, 291 (1966).

[54] K. Kawasaki, Ann. Phys. (N.Y.) 61, 1 (1970).

[55] L. Sjögren, Phys. Rev. A 22, 2866 (1980).

[56] W. Götze and L. Sjögren, Transp. Theory Stat. Phys. 24, 801 (1995).

[57] J.-L. Barrat, W. Götze, and A. Latz, J. Phys. Condens. Matter 1, 7163 (1989).

[58] M. Fuchs, W. Götze, S. Hildebrand, and A. Latz, Z. Phys. B 87, 43 (1992).

[59] M. Fuchs, I. Hofacker, and A. Latz, Phys. Rev. A 45, 898 (1992).

[60] M. Fuchs, Transp. Theory Stat. Phys. 24, 855 (1995).

[61] P. N. Pusey, in Liquids, Freezing and Glass Transition, edited by J. P. Hansen, D. Levesque, and J. Zinn-Justin (NorthHolland, Amsterdam, 1991), p. 763.

[62] G. Szamel and H. Löwen, Phys. Rev. A 44, 8215 (1991).

[63] K. Kawasaki, Physica A 208, 35 (1994).

[64] S. F. Edwards and P. W. Anderson, J. Phys. F 5, 965 (1975).

[65] F. R. Gantmacher, The Theory of Matrices (Chelsea, New York, 1960), Vol. II.

[66] V. I. Arnol'd, Catastrophe Theory, 3rd ed. (Springer-Verlag, Berlin, 1992).

[67] R. D. Mountain, J. Res. Natl. Bur. Stand. Sec. A 70, 207 (1966).

[68] W. Götze, in Amorphous and Liquid Materials, NATO Advanced Study Institute, Series E: Applied Physics, edited by E. Lüscher, G. Fritsch, and G. Jacucci (Martinus Nijhoff, Dordrecht, 1987), p. 34.

[69] S.-N. Chow and J. K. Hale, Methods of Bifurcation Theory (Springer-Verlag, Berlin, 1982).

[70] W. Götze, J. Phys. Condens. Matter 2, 8485 (1990). 
[71] W. Knaak, in Dynamics of Disordered Materials, edited by D. Richter, A. Dianoux, W. Petry, and J. Teixeira (SpringerVerlag, Berlin, 1989), p. 64.

[72] W. Kob and H. C. Andersen, Phys. Rev. E 52, 4134 (1995).

[73] J.-L. Barrat and A. Latz, J. Phys. Condens. Matter 2, 4289 (1990).

[74] S. P. Das and G. F. Mazenko, Phys. Rev. A 34, 2265 (1986).

[75] W. Götze and L. Sjögren, Z. Phys. B 65, 415 (1987).
[76] M. Fuchs, W. Götze, S. Hildebrand, and A. Latz, J. Phys. Condens. Matter 4, 7709 (1992).

[77] J. Baschnagel and M. Fuchs, J. Phys. Condens. Matter 7, 6761 (1995).

[78] H. Z. Cummins, W. M. Du, M. Fuchs, W. Götze, S. Hildebrand, A. Latz, G. Li, and N. J. Tao, Phys. Rev. E 47, 4223 (1993). 